\title{
Holographic subregion complexity for singular surfaces
}

\author{
Elaheh Bakhshaei $^{1}$, Ali Mollabashi ${ }^{2, a}$, Ahmad Shirzad ${ }^{1,3}$ \\ ${ }^{1}$ Department of Physics, Isfahan University of Technology, P.O.Box 84156-83111, Isfahan, Iran \\ ${ }^{2}$ School of Physics, Institute for Research in Fundamental Sciences (IPM), P.O.Box 19395-5531, Tehran, Iran \\ ${ }^{3}$ School of Particles and Accelerators, Institute for Research in Fundamental Sciences (IPM), P.O.Box 19395-5531, Tehran, Iran
}

Received: 24 March 2017 / Accepted: 21 September 2017 / Published online: 5 October 2017

(C) The Author(s) 2017. This article is an open access publication

\begin{abstract}
Recently holographic prescriptions were proposed to compute the quantum complexity of a given state in the boundary theory. A specific proposal known as 'holographic subregion complexity' is supposed to calculate the complexity of a reduced density matrix corresponding to a static subregion. We study different families of singular subregions in the dual field theory and find the divergence structure and universal terms of holographic subregion complexity for these singular surfaces. We find that there are new universal terms, logarithmic in the UV cut-off, due to the singularities of a family of surfaces including a kink in $(2$ $+1)$ dimensions and cones in even dimensional field theories. We also find examples of new divergent terms such as squared logarithm and negative powers times the logarithm of the UV cut-off parameter.
\end{abstract}

\section{Contents}

1 Introduction . . . . . . . . . . . . . 1

2 Singular subregions and summary of results ... . 2

Summary of results . . . . . . . . . . . . . . . 3

3 Flat locus singular surfaces . . . . . . . . . . . 4

3.1 Kink $k \ldots \ldots \ldots \ldots \ldots \ldots \ldots$

3.2 Cone $c_{n} \ldots \ldots \ldots \ldots \ldots \ldots$

3.3 Crease $k \times R^{m} \ldots \ldots \ldots \ldots$

3.4 Conical crease $c_{n} \times R^{m} \ldots \ldots \ldots$. . . . 9

4 Curved locus singular surfaces . . . . . . . . . . . 10

4.1 Crease $k \times \Sigma \ldots \ldots \ldots \ldots$

4.2 Conical crease $c_{n} \times \Sigma \ldots \ldots \ldots 14$

5 Discussions . . . . . . . . . . . . . . 15

References . . . . . . . . . . . . . . 16

a e-mail: mollabashi@ipm.ir

\section{Introduction}

Quantum entanglement has been widely studied in the context of holographic field theories after the pioneering proposal of Ryu-Takayanagi (RT) [1,2]. Quantum complexity is another notion in quantum information theory which has been recently included in the context of holographic field theories. Roughly speaking, the quantum complexity of a state is the minimum number of information gates needed to prepare a state from a given reference state. There have been made some efforts to develop a holographic dual for quantities related to this notion in the context of AdS/CFT correspondence [3-11].

From a more geometrical point of view, it is well established that the von Neumann entropy of a subregion in a given state corresponds to the area of a co-dimension two surface in the gravity solution dual of the state. One has also tried to find geometrical duals for other quantities in the context of information theory; such as Renyi entropies [12,13], information metric (fidelity susceptibility) $[14,18,19],{ }^{1}$ Fisher information [20], etc. Some of these geometrical objects are still co-dimension two objects in the dual theory but some are not.

There are two distinct proposals to compute complexity of a state in the dual gravity theory. The first one, which is sometimes called the 'complexity = volume' proposal, states that the complexity of a given state at a given time in the boundary theory is given by the volume of an extremal co-dimension one surface in the bulk which meets the corresponding time slice. To be more concrete, one can state this proposal as

$\mathcal{C}_{\mathrm{V}}=\max \left[\frac{V}{G_{N} \ell}\right]$,

where the maximum is chosen among those co-dimension one surfaces which end on the corresponding time slice on the conformal boundary. In this proposal $\ell$ is some length scale

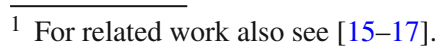


which should be identified case by case, e.g. the radius of the asymptotically AdS solution or the radius of the horizon in the case of AdS black-hole geometries. This non-recognized length scale seems to be a disadvantage of this proposal.

The other proposal, which is sometimes called 'complexity = action', states that the complexity of a given state at a given time is equal to the on-shell action of the dual (Einstein) gravity theory computed in the domain of dependence of any Cauchy surface in the bulk which ends on the given time slice at the conformal boundary. ${ }^{2}$ This region is known as the Wheeler-DeWitt patch, corresponding to the given boundary time slice. Although this proposal (in contrast with the previous one) does not need any length scale by definition, it has its own challenges due to surface terms and corner contributions of the Wheeler-DeWitt patch (see [11,22]). We will come back to this point in the next section.

A natural generalization of the 'complexity = volume' proposal concerns with generic mixed states. A specific way of constructing a mixed state out of the entire state of a system is to trace out a part of the space-like manifold of the dual field theory. The mixed state constructed in this way is described by what is known as the reduced density matrix. Then the complexity of such a (static) state is proposed to be given by the volume enclosed by the Ryu-Takayanagi surface and the corresponding subregion in the boundary theory. ${ }^{3}$ To be more concrete the subregion complexity is defined as [18]

$\mathcal{C}_{\text {subregion }}=\frac{V(\gamma)}{8 \pi \ell G_{N}}$

where $\gamma$ is the RT surface of the corresponding subregion and $\ell$ is a length scale of the dual geometry. This proposal (up to a numerical factor) reduces to 'complexity = volume' given in (1.1) if the subregion is chosen to be the whole time slice of the dual theory.

Different proposals for complexity all lead to UV divergent results since they all contain a volume of a surface which reaches the conformal boundary of an asymptotically AdS geometry. This is the same as what happened in the case of holographic entanglement entropy. ${ }^{4}$ Natural questions as regards such quantities are: What is the divergent structure of this quantity? How it can be regularized? What kind of universal information can be extracted from it? Is it possible to find any monotonic function out of this quantity under the RG flow of the dual theory? ${ }^{5}$ Specifically for the case of subregion complexity one may also question the (subregion) shape dependence of the divergence structure.

\footnotetext{
${ }^{2}$ Recently some progress has been made for complexity in higher derivative theories in [21].

${ }^{3}$ Recently a covariant generalization of this proposal is given in [11].

${ }^{4}$ A concrete example of a dual CFT calculation for Fischer information metric in the case of marginal deformations has been presented in [14].

${ }^{5}$ See [23] for a related case study.
}

Some of the above questions have been recently addressed for different proposals of complexity and even for the complexity of reduced states due to smooth subregions [11]. The goal of this paper is to investigate the divergence structure of subregion complexity when the subregion is a singular surface. Similar to the case of entanglement entropy we expect new divergent (sometimes new universal) terms due to singularities in the subregion. There have been made a considerable amount of efforts investigating the role of singularities of entangling regions in the context of (mostly holographic) entanglement entropy [24-37]. In the case of entanglement entropy it has been shown that in the smooth limit beyond the leading order one can give some information as regards the four point function of the stress tensor [38].

We will consider the simplest case of a singular surface in a $(2+1)$-dimensional field theory and its generalizations to sufficiently symmetric singular surfaces in higher dimensions (see $[27,28]$ for a similar analysis for entanglement entropy) and we study the divergent structure due to subregion complexity proposal [18].

The rest of this paper is organized as follows: in Sect. 2 we define different families of the singular surfaces which we study. If the reader is just interested in the final results, we have summarized our subsequent results in this section. In the following sections we study complexity of different subregions and we finally in the last section address interesting directions for future studies.

\section{Singular subregions and summary of results}

We are interested in asymptotically AdS solutions of Einstein gravity with a negative cosmological constant in $d+1$ dimensions. The simplest case which we study in this section is the pure $A d S_{d+1}$ solution in the Poincaré patch with the following coordinates:

$$
\begin{aligned}
\mathrm{d} s^{2}= & \frac{L^{2}}{z^{2}}\left[-\mathrm{d} t^{2}+\mathrm{d} z^{2}+\mathrm{d} \rho^{2}\right. \\
& \left.+\rho^{2}\left(\mathrm{~d} \theta^{2}+\sin ^{2} \theta \mathrm{d} \Omega_{n}^{2}\right)+\sum_{i=1}^{m}\left(\mathrm{~d} x^{i}\right)^{2}\right]
\end{aligned}
$$

where $z$ is the radial coordinate and $L$ is the AdS radius. Here $\mathrm{d} \theta^{2}+\sin ^{2} \theta \mathrm{d} \Omega_{n}^{2}$ is the metric on a unit sphere $S_{n+1}$ and the term $\sum_{i=1}^{m}\left(\mathrm{~d} x^{i}\right)^{2}$ indicates a flat $R^{m}$ space in Cartesian coordinates. The conformal boundary of this solution is achieved in the $z \rightarrow 0$ limit. Hence, the boundary metric reads

$$
\begin{aligned}
\mathrm{d} s^{2}= & -\mathrm{d} t^{2}+\mathrm{d} \rho^{2}+\rho^{2}\left(\mathrm{~d} \theta^{2}+\sin ^{2} \theta \mathrm{d} \Omega_{n}^{2}\right) \\
& +\sum_{i=1}^{m}\left(\mathrm{~d} x^{i}\right)^{2} .
\end{aligned}
$$


For the whole manifold of the bulk, as well as the boundary, the range of the parameter $\theta$ is $(-\pi, \pi)$ for $n=0$ and $(0, \pi)$ for $n>0$. However, throughout this paper we consider different kinds of singular subregions, i.e. the conic singular subregions, in which $-\Omega<\theta<\Omega$ for $n=0$ and $0<\theta<\Omega$ for $n>0$. The simplest conical geometry is a kink $(k)$ in $d=3$ where $n=m=0$, as the following subregion of the boundary:

$k=\left\{t_{E}=0, \rho=[0, \infty),-\Omega<\theta<\Omega\right\}$.

The cone family $\left(c_{n}\right)$ of singular surfaces in $d=n+3$ dimensions consists of manifolds with $m=0$ and $n \geq 1$ in Eq. (2.2) confined to the region

$c_{n}=\left\{t_{E}=0, \rho=[0, \infty), \theta=\Omega\right\}$.

The crease family in $d=3+m$ dimensions is the manifold $\left(k \times R^{m}\right)$ derived by considering $n=0$ and $m \geq 1$ in Eq. (2.1). We also consider mixed cases where both integers $n$ and $m$ are non-zero which we call them cone-crease.

We also study singular surfaces in asymptotically $\operatorname{AdS}_{d+1}$ geometries given by

$$
\begin{aligned}
\mathrm{d} s^{2}= & \frac{L^{2}}{z^{2}}\left[\mathrm{~d} z^{2}+f_{1}(z)\left(-\mathrm{d} t^{2}+\mathrm{d} \rho^{2}\right.\right. \\
& \left.\left.+\rho^{2}\left(\mathrm{~d} \theta^{2}+\sin ^{2} \theta \mathrm{d} \Omega_{n}^{2}\right)\right)+f_{2}(z) R^{2} \mathrm{~d} \Omega_{m}^{2}\right] .
\end{aligned}
$$

also some specific higher derivative gravity theories. In this paper we calculate the holographic complexity in each case by using the proposal of Ref. [18]. As we have mentioned in the previous section, according to this proposal the volume of a co-dimension one surface enclosed by the subregion in the boundary theory and the RT co-dimension two surface in the bulk is proportional to the complexity of the (mixed) state corresponding to the subregion. To do so, one should find the RT surface corresponding to subregion $A$, which we denote by $\gamma_{A}$, and calculate the volume $V\left(\gamma_{A}\right)$ enclosed by $\gamma_{A}$. The holographic complexity given by Eq. (1.2) is proposed [18]. We choose $\ell$ in the asymptotically AdS gravity solutions to be identified with the AdS radius. In what follows we will study this quantity in different singular subregions.

Summary of results

Since the detailed calculations presented in the following sections may be involved, here we briefly summarize our results. We study the divergent structure of holographic subregion complexity and find new divergences due to singular subregions which in some cases lead to new universal terms.

In the case of a crease entangling region in a $(2+1)$ dimensional boundary theory (see the left panel of Fig. 1) we find that there is a new divergent term of the form $\log \delta$, which is a universal term. The entanglement entropy for the same subregion also leads to a logarithmic universal term.

\begin{tabular}{llllll}
\hline$d$ & $\begin{array}{l}\text { Background } \\
\text { space-time }\end{array}$ & $\begin{array}{l}\text { Geometry of } \\
\text { entangling surface }\end{array}$ & $\begin{array}{l}\text { Crease } \\
\text { dimension }\end{array}$ & $\begin{array}{l}\text { Expected } \\
\text { divergences }\end{array}$ & $\begin{array}{l}\text { New } \\
\text { divergences }\end{array}$ \\
\hline 3 & $R^{3}$ & $k$ & 0 & $1 / \delta^{2}$ & $\log \delta$ \\
4 & $R^{4}$ & $c_{1}$ & 0 & $1 / \delta^{3}, 1 / \delta$ & $\log \delta$ \\
5 & $R^{5}$ & $c_{2}$ & 0 & $1 / \delta^{4}, 1 / \delta^{2}, \log \delta$ & $\log ^{2} \delta$ \\
6 & $R^{6}$ & $c_{3}$ & 0 & $1 / \delta^{5}, 1 / \delta^{3}, 1 / \delta$ & $\log ^{2} \delta$ \\
7 & $R^{7}$ & $k$ & $1 / \delta^{6}, 1 / \delta^{4}, 1 / \delta^{2}, \log \delta$ & $\log ^{2} \delta$ \\
$>3$ & $R^{d}$ & $k \times R^{d-3}$ & $1-3$ & $1 / \delta^{d-1}, 1 / \delta^{d-3}$ & - \\
4 & $R^{3} \times S^{1}$ & $k \times S^{1}$ & 1 & $1 / \delta^{3}$ & - \\
5 & $R^{3} \times S^{2}$ & $k \times S^{3}$ & 2 & $1 / \delta^{4}, 1 / \delta^{2}, \log \delta$ & - \\
6 & $R^{3} \times S^{3}$ & $k \times\left(R^{1} \times S^{2}\right)$ & 3 & $1 / \delta^{5}, 1 / \delta^{3}$ & - \\
6 & $R^{4} \times S^{2}$ & $c_{1} \times R^{1}$ & $1 / \delta^{5}, 1 / \delta^{3}, 1 / \delta$ & - \\
5 & $R^{5}$ & $c_{1} \times R^{2}$ & 2 & $1 / \delta^{4}, 1 / \delta^{2}, \log \delta$ & $1 / \delta$ \\
6 & $R^{6}$ & $c_{1} \times S^{1}$ & 1 & $1 / \delta^{5}, 1 / \delta^{3}, 1 / \delta$ & $1 / \delta^{2}$ \\
5 & $R^{4} \times S^{1}$ & $c_{1} \times S^{2}$ & $1 / \delta^{4}, 1 / \delta^{2}, \log \delta$ & $1 / \delta$ \\
6 & $R^{4} \times S^{2}$ & $c_{2} \times R^{1}$ & 2 & $1 / \delta^{5}, 1 / \delta^{3}, 1 / \delta$ & $1 / \delta^{2}, \log \delta$ \\
6 & $R^{6}$ & 1 & $1 / \delta^{5}, 1 / \delta^{3}, 1 / \delta$ & $1 / \delta \log \delta$ \\
7 & $R^{7}$ & $c_{2} \times R^{2}$ & 2 & $1 / \delta^{6}, 1 / \delta^{4}, 1 / \delta^{2}, \log \delta$ & $1 / \delta^{2} \log \delta$ \\
6 & $R^{5} \times S^{1}$ & 1 & $1 / \delta^{5}, 1 / \delta^{3}, 1 / \delta$ & $1 / \delta \log \delta$ \\
\hline
\end{tabular}

In these cases $f_{1}$ and $f_{2}$ are functions which are determined by the gravity equations of motion. We study different cones and creases in these asymptotically AdS geometries.

In Ref. [27] the holographic entanglement entropy for the above singular surfaces is calculated in Einstein gravity and
For the case of a crease entangling region with a flat locus, which we denote by $k \times R^{m}$ (see the right panel of Fig. 1), there is no universal term due to the singularity and even no actual new divergent term, although the subleading divergent term gets corrections from the singularity. This resembles the entanglement entropy in having no new universal term. 

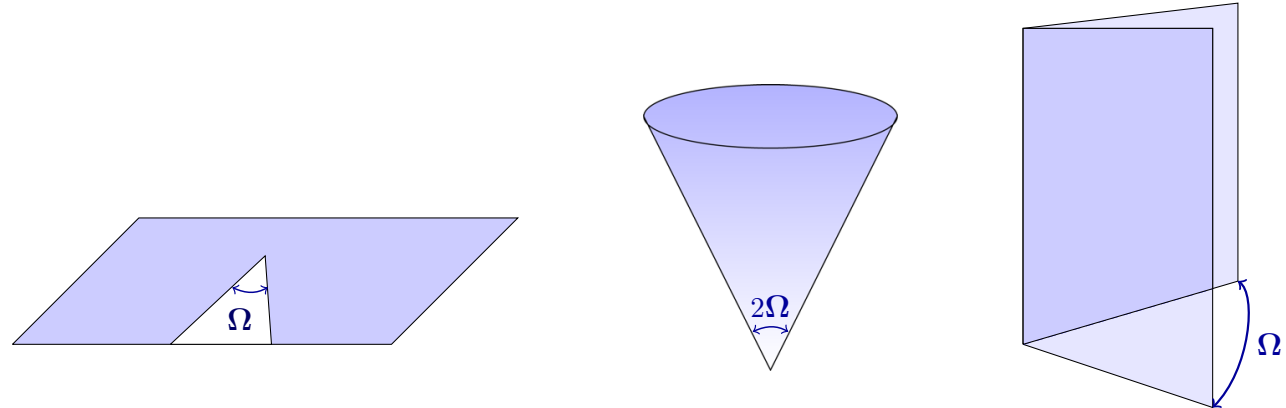

Fig. 1 Left: The blue plane represents a constant time slice of a $d=3$ CFT with a kink $(k)$ entangling region on it. Middle: Conical entangling region in a $d=4$ CFT. Right: A crease $\left(k \times R^{m}\right)$ entangling region as a direct generalization of the kink in higher dimensions

Even for the case of $k \times S^{1}$, for which again the locus of the singularity is flat, there is no new universal term and no new divergent contribution from the singularity.

In the case of creases with a curved locus we again find that there is no new divergent term. This is in contrast with what happens for entanglement entropy of these surfaces. We study the case of $k \times S^{2}$ and $k \times S^{3}$ and also $k \times R \times S^{2}$ and in all of them, although there is a $\log \delta$ term, it is suppressed with a positive power of $\delta$, resulting in no new divergent term.

The most interesting behavior happens for conical subregions which we show by $c_{n}$ (see the middle panel of Fig. 1). For these subregions we find that there is a new universal $\log \delta$ term for odd $n$ and $\log ^{2} \delta$ for even $n$. We have worked out a few examples for $n=1,2,3,4$. In comparison with the entanglement entropy of these surfaces we find a shift from odd to even $n$ where $\log ^{2} \delta$ and $\log \delta$ appear, respectively. It would be very interesting to see whether these universal terms are related to some characteristic feature of the dual field theory.

The other family of singular surfaces which we have studied are conical creases of the form $c_{n} \times R^{m}$ and $c_{n} \times S^{m}$. Among these surfaces the only case where we find that a universal $\log \delta$ term appears is $c_{1} \times S^{2}$. In other cases new divergent terms appear due to the singularity which has the form of $1 / \delta \log \delta$ or $1 / \delta^{2} \log \delta$. These are very similar to what has been recently found from the 'complexity $=$ action' proposal [11]. This similarity may be due to the singularities within the Wheeler-DeWitt patch. We have summarized our results in the table.

\section{Flat locus singular surfaces}

\subsection{Kink $k$}

The simplest case is a kink in a $2+1$ dimensional boundary theory. The bulk metric dual to the vacuum state is given by

$\mathrm{d} s^{2}=\frac{L^{2}}{z^{2}}\left(-\mathrm{d} t^{2}+\mathrm{d} z^{2}+\mathrm{d} \rho^{2}+\rho^{2} \mathrm{~d} \theta^{2}\right)$, and the subregion in defined in constant time slice as $\rho \in$ $[0, H]$ and $\theta \in[-\Omega, \Omega]$, where $H$ is an IR cut-off. The corresponding Ryu-Takayanagi surface can be described by $z=z(\rho, \theta)$; hence the entanglement entropy is given by

$S_{3}^{\text {kink }}=\frac{2 \pi L^{2}}{l_{p}^{2}} \int \mathrm{d} \rho \mathrm{d} \theta \frac{\sqrt{\rho^{2}+\rho^{2} z^{\prime 2}+\dot{z}^{2}}}{z^{2}}$,

where $z^{\prime}=\partial_{\rho} z$ and $\dot{z}=\partial_{\theta} z$. Since there is no length scale except $\rho$, the radial coordinate $z$ depends on $\rho$ linearly [26], i.e.

$z=\rho h(\theta)$,

and $h(\theta)$ should be found such that it minimizes the entropy (area) functional and is anchored to the kink in the asymptotic boundary. Applying this to Eq. (3.2) gives

$S_{3, k}=\frac{4 \pi L^{2}}{l_{p}^{2}} \int_{\delta / h_{0}}^{H} \frac{\mathrm{d} \rho}{\rho} \int_{0}^{\Omega-\epsilon} \mathrm{d} \theta \frac{\sqrt{1+h^{2}+\dot{h}^{2}}}{h^{2}}$,

where $\dot{h}=\mathrm{d} h / \mathrm{d} \theta, h(0)=h_{0}$ and $z=\delta$ is UV cut-off. However, since the integrand of Eq. (3.4) does not depend on $\theta$ explicitly, we have the following conserved quantity along the $\theta$ translation:

$K=\frac{\left(1+h^{2}\right)}{h^{2} \sqrt{1+h^{2}+\dot{h}^{2}}}=\frac{\sqrt{1+h_{0}^{2}}}{h_{0}^{2}}$.

To find the holographic subregion complexity we should write the volume $V(\gamma)$ of the subregion of the bulk,

$$
\begin{aligned}
V(\gamma) & =L^{3} \int_{\delta / h_{0}}^{H} \mathrm{~d} \rho \rho \int_{-\Omega+\varepsilon}^{\Omega-\varepsilon} \mathrm{d} \theta \int_{\delta}^{z} \frac{\mathrm{d} z}{z^{3}} \\
& =\frac{L^{3}}{2} \int_{\delta / h_{0}}^{H} \mathrm{~d} \rho \rho \int_{-\Omega+\varepsilon}^{\Omega-\varepsilon} \mathrm{d} \theta\left(\frac{1}{\delta^{2}}-\frac{1}{z^{2}}\right) \\
& =\frac{\Omega L^{3}}{2 \delta^{2}}\left(H^{2}-\frac{\delta^{2}}{h_{0}^{2}}\right)-L^{3} \int_{\delta / h_{0}}^{H} \frac{\mathrm{d} \rho}{\rho} \int_{0}^{\Omega-\varepsilon} \frac{\mathrm{d} \theta}{h^{2}},
\end{aligned}
$$



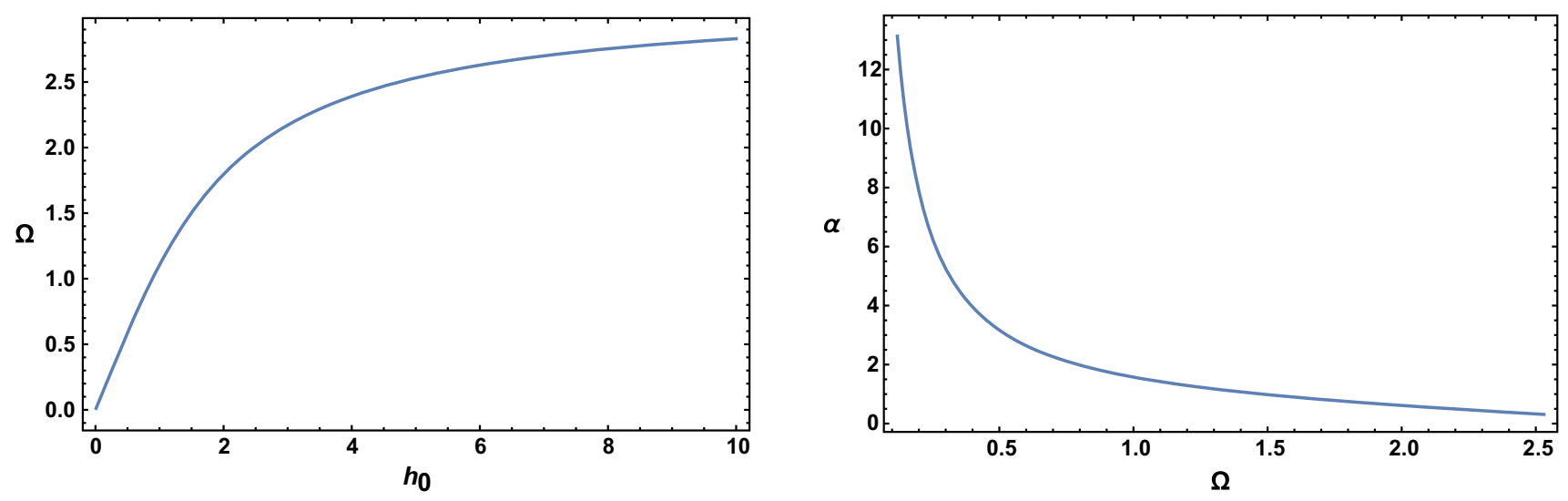

Fig. 2 Right panel: The opening angle $\Omega$ is plotted versus the turning point $h_{0}$. Left panel: The coefficient of the universal (logarithmic) term of complexity for a kink is plotted versus the boundary data $\Omega$

where $\epsilon$ is a short distance cut-off in the boundary corresponding to $\delta$ in the bulk. To clarify the singular terms of Eq. (3.6) we convert $\theta$ integration to an integral over $h$ as follows:

$\int_{0}^{\Omega-\varepsilon} \frac{\mathrm{d} \theta}{h^{2}}=\int_{h_{0}}^{\delta / \rho} \frac{\mathrm{d} h}{h^{2} \dot{h}}$.

One can easily find the following expression from Eq. (3.5):

$\dot{h}=-\sqrt{\frac{\left(1+h^{2}\right)^{2} h_{0}^{4}-h^{4}\left(1+h_{0}^{2}\right)\left(1+h^{2}\right)}{h^{4}\left(1+h_{0}^{2}\right)}}$.

Using the coordinate transformation $y=\sqrt{\frac{1}{h^{2}}-\frac{1}{h_{0}^{2}}}$, where $y \rightarrow \infty$ as we approach the boundary via $\theta \rightarrow \Omega$, we have

$$
\begin{aligned}
& \int_{h_{0}}^{\delta / \rho} \frac{\mathrm{d} h}{h^{2} \dot{h}}=\int_{0}^{\sqrt{(\rho / \delta)^{2}-1 / h_{0}^{2}}} \mathrm{~d} y \\
& \quad \times \sqrt{\frac{\left(1+h_{0}^{2}\right)}{\left(1+h_{0}^{2}+y^{2} h_{0}^{2}\right)\left(2+h_{0}^{2}+y^{2} h_{0}^{2}\right)}} .
\end{aligned}
$$

In the limit $\delta \rightarrow 0$ and hence $y \rightarrow \infty$ the integrand is finite. So we can find it just for $y \rightarrow \infty$. We have finally

$V(\gamma)=L^{3}\left[\frac{\Omega}{2} \frac{H^{2}}{\delta^{2}}+\alpha\left(h_{0}\right) \log \left(\frac{\delta}{H}\right)\right]+$ finite,

where $\alpha\left(h_{0}\right)$ is the cut-off independent term given by

$\alpha\left(h_{0}\right)=\int_{0}^{\infty} d y \sqrt{\frac{\left(1+h_{0}^{2}\right)}{\left(1+h_{0}^{2}+y^{2} h_{0}^{2}\right)\left(2+h_{0}^{2}+y^{2} h_{0}^{2}\right)}}$,

which vanishes in the smooth region limit (i.e. $\Omega \rightarrow \pi$ ). Thus the divergent structure of the holographic complexity of the kink is given by
$\mathcal{C}_{k}=\frac{L^{2}}{8 \pi G_{N}}\left[\frac{\Omega}{2} \frac{H^{2}}{\delta^{2}}+\alpha\left(h_{0}\right) \log \left(\frac{\delta}{H}\right)\right]$.

In Fig. 2 we have plotted $\alpha$ as a function of the opening angle of the kink $\Omega$. As expected our numerical result shows that in the smooth limit, that is, $\Omega \rightarrow \pi$, the new universal term vanishes.

\subsection{Cone $c_{n}$}

As indicated in the previous section, to consider a conical subregion $c_{n}$ with $n=d-3$, we use the following form of the bulk metric:

$\mathrm{d} s^{2}=\frac{L^{2}}{z^{2}}\left[-\mathrm{d} t^{2}+\mathrm{d} z^{2}+\mathrm{d} \rho^{2}+\rho^{2}\left(\mathrm{~d} \theta^{2}+\sin ^{2} \theta \mathrm{d} \Omega_{n}^{2}\right)\right]$,

where $\mathrm{d} \Omega_{n}$ is the metric of a unit sphere $S_{n}$. The subregion in the boundary is defined by $\rho \in[0, H]$ and $\theta \in[0, \Omega]$. The extension of this region in the bulk is denoted by the function $z(\rho, \theta)$. One should find the profile of this extension via minimizing the following area functional:

$$
\begin{aligned}
S= & L^{d-1} \Omega_{n} \int \mathrm{d} \rho \mathrm{d} \theta \frac{\rho^{d-3}}{z^{d-1}} \\
& \times \sin ^{d-3} \theta \sqrt{\rho^{2}+\rho^{2} z^{\prime 2}+\dot{z}^{2}},
\end{aligned}
$$

where $\Omega_{n}$ is the volume of the unit $n$-sphere and $\dot{z}=\partial_{\theta} z, z^{\prime}=$ $\partial_{\rho} z$.

As in the previous case, $z$ can depend on $\rho$ only linearly, i.e. $z(\rho, \theta)=\rho h(\theta)$. Using this assumption and the change of variable $y=\sin \theta=y(h)$, which gives

$\dot{h}=\frac{\sqrt{1-y^{2}}}{y^{\prime}}, \quad \ddot{h}=-\frac{y y^{\prime 2}+\left(1-y^{2}\right) y^{\prime \prime}}{y^{\prime 3}}$,

the equation of motion for the case $d=4$ reads as follows: 


$$
\begin{aligned}
0= & h\left(1+h^{2}\right) y\left(1-y^{2}\right) y^{\prime \prime} \\
& -y y^{\prime}\left(3+h^{2}+\left(3+5 h^{2}+2 h^{4}\right) y^{\prime 2}\right) \\
& +2 h y^{2}\left(1+\left(1+h^{2}\right) y^{\prime 2}\right)-h\left(1+\left(1+h^{2}\right) y^{\prime 2}\right) \\
& +\left(3+h^{2}\right) y^{3} y^{\prime}-h y^{4},
\end{aligned}
$$

where $y^{\prime}=\frac{\mathrm{d} y}{\mathrm{~d} h}$ and $y^{\prime \prime}=\frac{d^{2} y}{\mathrm{~d} h^{2}}$. Since we are interested in the singular behavior of the complexity near the boundary, where $h \rightarrow 0$, let us concentrate on this limit (still for $d=4$ ). For this reason we consider a power law expansion for $y(h)$ in terms of $h$ and put it in Eq. (3.15). Then using the boundary condition $y(0)=\sin \Omega$ we find the following result:

$y=\sin (\Omega)-\frac{1}{4} \cos (\Omega) \cot (\Omega) h^{2}+\mathcal{O}\left(h^{4}\right)$.

The expansion for $\dot{h}$ follows consequently from $\dot{h}=$ $\frac{\sqrt{1-y^{2}}}{y^{\prime}(h)}$ as

$\dot{h}=-\frac{2 \tan (\Omega)}{h}-\frac{1}{2} h(3-\cos (2 \Omega)) \csc (2 \Omega) \log (h)+\mathcal{O}(h)$.

The corresponding volume is given by

$$
\begin{aligned}
V(\gamma)= & L^{4} \Omega_{1} \int \mathrm{d} \rho \rho^{2} \int \mathrm{d} \theta \sin (\theta) \int_{\delta}^{z} \frac{\mathrm{d} z}{z^{4}} \\
= & \frac{L^{4}}{3} \Omega_{1} \int \mathrm{d} \rho \rho^{2} \int \mathrm{d} \theta \sin (\theta)\left(\frac{1}{\delta^{3}}-\frac{1}{z^{3}}\right) \\
= & \frac{2 \pi L^{4} H^{3}}{9 \delta^{3}}(1-\cos (\Omega)) \\
& -\frac{L^{4} 2 \pi}{3} \int_{\delta / h_{0}}^{H} \frac{\mathrm{d} \rho}{\rho} \int_{h_{0}}^{\delta / \rho} \mathrm{d} h \frac{\sin (\theta)}{h^{3} \dot{h}}+\text { finite. }
\end{aligned}
$$

Using asymptotic expansions (3.16) and (3.17) the integrand of (3.18) has the following behavior near the boundary:

$$
\begin{aligned}
\frac{\sin (\theta)}{h^{3} \dot{h}} \sim & -\frac{1}{2} \frac{\cos (\Omega)}{h^{2}} \\
& +\frac{1}{8} \cot ^{2}(\Omega) \sin (\Omega) \csc (2 \Omega)(3-\cos (2 \Omega)) \log (h) \\
& +\frac{1}{8} \cos (\Omega) \cot ^{2}(\Omega)+\mathcal{O}(h)
\end{aligned}
$$

Let us divide the singular parts of $V(\gamma)$ into $I_{1}$ and $L_{2}$ where the latter contains the singularities due to the integrand while the former shows the contribution of the limits of the integrations, i.e.

$$
\begin{aligned}
I_{1}= & -\frac{L^{4} 2 \pi}{3} \int_{\delta / h_{0}}^{H} \frac{\mathrm{d} \rho}{\rho} \int_{h_{0}}^{\delta / \rho} \mathrm{d} h\left[\frac{\sin (\theta)}{h^{3} \dot{h}}+\frac{1}{2} \frac{\cos (\Omega)}{h^{2}}\right. \\
& \times \frac{1}{8} \cot ^{2}(\Omega) \sin (\Omega) \csc (2 \Omega)
\end{aligned}
$$

$$
\begin{aligned}
& \left.\times(3-\cos (2 \Omega)) \log (h)-\frac{1}{8} \cos (\Omega) \cot ^{2}(\Omega)\right], \\
I_{2}= & \frac{2 \pi L^{4} H^{3}}{9 \delta^{3}}(1-\cos (\Omega))-\frac{L^{4} 2 \pi}{3} \int_{\delta / h_{0}}^{H} \frac{\mathrm{d} \rho}{\rho} \int_{h_{0}}^{\delta / \rho} \\
& \times \mathrm{d} h\left[-\frac{1}{2} \frac{\cos (\Omega)}{h^{2}}+\frac{1}{8} \cot ^{2}(\Omega) \sin (\Omega) \csc (2 \Omega)\right. \\
& \left.\times(3-\cos (2 \Omega)) \log (h)+\frac{1}{8} \cos (\Omega) \cot ^{2}(\Omega)\right] .
\end{aligned}
$$

So the singular part of the complexity is given by

$\mathcal{C}_{4, c_{1}}=\frac{1}{8 \pi L G_{N}}\left(I_{1}+I_{2}\right)$.

In the limit $h \rightarrow \delta / \rho$ there is no singular term from integration over $h$ (neither from the integrand nor from the integration limits); we have just a logarithmic singularity from the lower limit of the integration over $\rho$ as follows:

$$
\begin{aligned}
I_{1}= & \frac{L^{4} 2 \pi}{3} \log \delta \int_{h_{0}}^{0} \mathrm{~d} h\left(\frac{\sin (\theta)}{h^{3} \dot{h}}+\frac{1}{2} \frac{\cos (\Omega)}{h^{2}}\right. \\
& -\frac{1}{8} \cot ^{2}(\Omega) \sin (\Omega) \csc (2 \Omega)(3-\cos (2 \Omega)) \log (h) \\
& \left.-\frac{1}{8} \cos (\Omega) \cot ^{2}(\Omega)\right) .
\end{aligned}
$$

The singular terms in $I_{2}$ can be calculated directly. Hence we have

$$
\begin{aligned}
\mathcal{C}_{4, c_{1}}= & \frac{L^{3}}{8 G_{N}}\left[\frac{2(1-\cos (\Omega))}{9} \frac{H^{3}}{\delta^{3}}\right. \\
& \left.-\frac{\cos (\Omega)}{3} \frac{H}{\delta}+\frac{\beta\left(h_{0}\right)}{3} \log \left(\frac{\delta}{H}\right)\right],
\end{aligned}
$$

where

$$
\begin{aligned}
\beta\left(h_{0}\right)= & 2 \int_{h_{0}}^{0} \mathrm{~d} h\left(\frac{\sin (\theta)}{h^{3} \dot{h}}+\frac{1}{2} \frac{\cos (\Omega)}{h^{2}}\right. \\
& -\frac{1}{8} \cot ^{2}(\Omega) \sin (\Omega) \csc (2 \Omega)(3-\cos (2 \Omega)) \log (h) \\
& \left.-\frac{1}{8} \cos (\Omega) \cot ^{2}(\Omega)\right) \\
& -\frac{\cos (\Omega)}{h_{0}}-\frac{h_{0}}{4} \cos (\Omega) \cot ^{2}(\Omega) \\
& +\frac{1}{4} h_{0}\left(1-\log \left(h_{0}\right)\right) \cot ^{2}(\Omega) \sin (\Omega) \\
& \times \csc (2 \Omega)(3-\cos (2 \Omega))
\end{aligned}
$$

In Fig. 3 we have plotted the turning point in terms of the opening angle for $c_{1}$. As expected in the smooth limit, that is, $\Omega \rightarrow \pi / 2$, the coefficient of the new divergent term vanishes. 

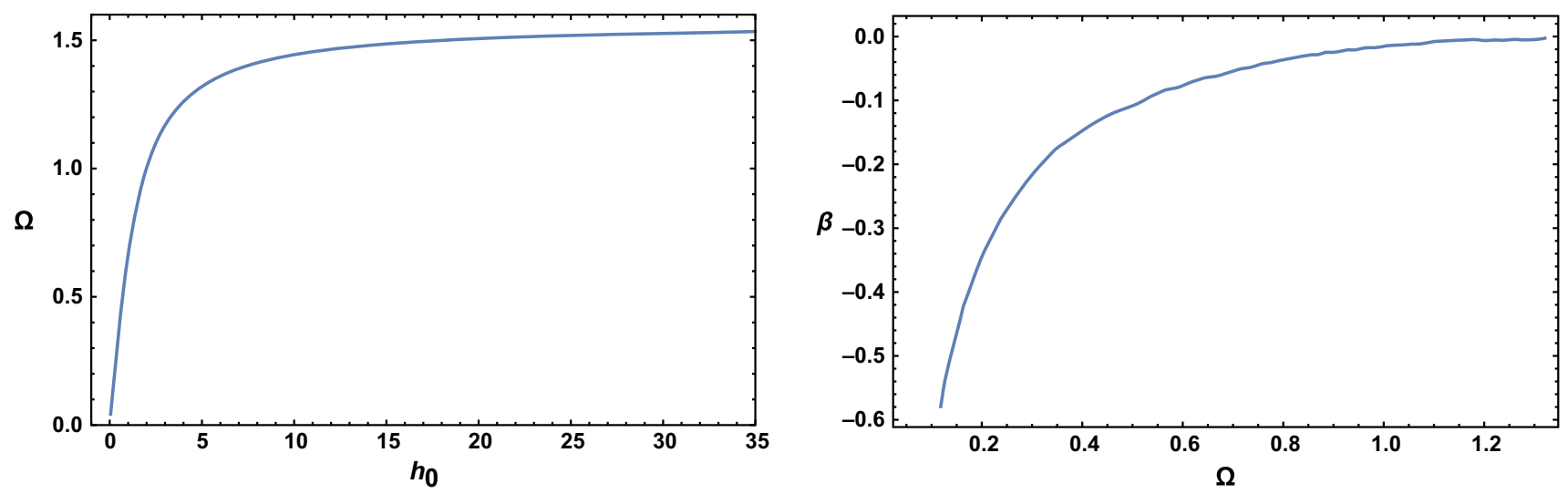

Fig. 3 Right panel: The opening angle $\Omega$ is plotted versus the turning point $h_{0}$ for a cone in $d=4$. Left panel: The coefficient of the universal (logarithmic) term of complexity is plotted versus the boundary data $\Omega$
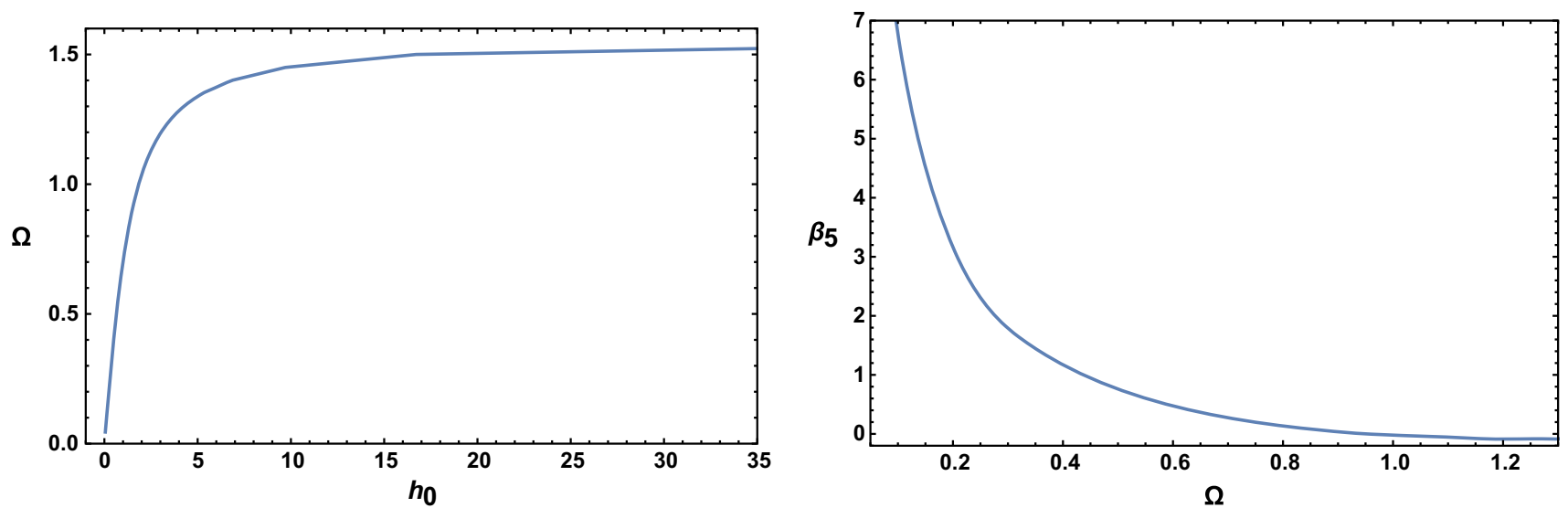

Fig. 4 Right panel: The opening angle $\Omega$ is plotted versus the turning point $h_{0}$ for a cone in $d=5$. Left panel: The coefficient of the universal term [first line of Eq. (3.26)] of complexity is plotted versus the boundary data $\Omega$

One can perform similar computations for cones in higher dimensions. We have done this for $c_{2}$ and $c_{3}$ in $\mathrm{CFT}_{5}$ and $\mathrm{CFT}_{6}$, respectively. The method is similar to what we have presented in $d=4$, so we will skip the details and report the results in these cases.

In the case of $c_{2}$ one finds two families of divergent terms proportional to $\log \delta$ and $\log ^{2} \delta$, which are given by

$$
\begin{aligned}
\mathcal{C}_{5, c_{2}}^{\log }= & \frac{L^{4}}{8 G_{N}} \log \left(\frac{\delta}{H}\right) \\
& \times\left(\int _ { h _ { 0 } } ^ { 0 } \mathrm { d } h \left[\frac{\sin ^{2}(\theta)}{h^{4} \dot{h}}-\frac{4 \cos ^{2}(\Omega) \cot (\Omega)}{9 h}\right.\right. \\
& \left.\left.+\frac{2 \cos (\Omega) \sin (\Omega)}{3 h^{3}}\right]-\frac{\cos (\Omega) \sin (\Omega)}{3 h_{0}^{2}}\right) \\
\mathcal{C}_{5, c_{2}}^{\log ^{2}}= & \frac{L^{4}}{36 G_{N}} \cos ^{2}(\Omega) \cot (\Omega) \log ^{2}\left(\frac{\delta}{H}\right) .
\end{aligned}
$$

One should note that $\mathcal{C}_{5, c_{2}}^{\log ^{2}}$ is not a universal term. In Fig. 4 we have plotted the turning point in terms of the opening angle for $c_{2}$. As expected in the smooth limit, that is, $\Omega \rightarrow \pi / 2$, the coefficient of the new divergent term vanishes.

For the case $c_{3}$ we find

$$
\begin{aligned}
\mathcal{C}_{6, c_{3}}^{\log }= & \frac{L^{5} \pi}{20 G_{N}} \log \left(\frac{\delta}{H}\right)\left[\int _ { h _ { 0 } } ^ { 0 } \mathrm { d } h \left(\frac{\sin ^{3}(\theta)}{h^{5} \dot{h}}+\frac{3}{4} \frac{\cos (\Omega) \sin ^{2}(\Omega)}{h^{4}}\right.\right. \\
& -\frac{3}{256} \frac{\cos (\Omega)(67+35 \cos (2 \Omega))}{h^{2}} \\
& -\frac{27}{8192}(155+106 \cos (2 \Omega) \\
& \left.\left.+15 \cos (4 \Omega)) \cot ^{2}(\Omega) \csc (\Omega)\right)\right) \\
& -\frac{\cos (\Omega) \sin ^{2}(\Omega)}{4 h_{0}^{3}}+\frac{3 \cos (\Omega)(67+35 \cos (2 \Omega)}{256 h_{0}} \\
& +\frac{27 h_{0}}{8192}(155+106 \cos (2 \Omega) \\
& \left.\left.+15 \cos (4 \Omega)) \cot ^{2}(\Omega) \csc (\Omega)\right)\right] .
\end{aligned}
$$




\subsection{Crease $k \times R^{m}$}

Consider the following metric for a $A d S_{d+1}$ space-time in the bulk:

$\mathrm{d} s^{2}=\frac{L^{2}}{z^{2}}\left[-\mathrm{d} t^{2}+\mathrm{d} z^{2}+\mathrm{d} \rho^{2}+\rho^{2} \mathrm{~d} \theta^{2}+\sum_{i=1}^{m}\left(\mathrm{~d} x^{i}\right)^{2}\right]$

where the Cartesian coordinates $x^{i}$ denote a $R^{m}$ flat space for $m=d-3$. Consider a kink subregion defined as $\theta \in[-\Omega, \Omega]$ and $\rho \in[0, \infty]$ for the full range of $x^{i} \in[-\infty, \infty]$. However, to avoid IR singularities in the following calculations we restrict ourselves to the limited region $\rho \in[0, H]$ and $x^{i} \in\left[-\frac{\tilde{H}}{2}, \frac{\tilde{H}}{2}\right]$. Assume that the extension of the entangling region in the bulk is given by the radial coordinate $z=z(\rho, \theta)$. Hence, the induced metric on the extended surface reads

$h=\left(\begin{array}{ccccc}\frac{L^{2}}{z^{2}}\left(1+\left(z^{\prime}\right)^{2}\right) & \frac{L^{2}}{z^{2}} z^{\prime} \dot{z} & & & \\ \frac{L^{2}}{z^{2}} z^{\prime} \dot{z} & \frac{L^{2}}{z^{2}}\left(\rho^{2}+\dot{z}^{2}\right) & & \\ & & \frac{L^{2}}{z^{2}} & \\ & & & \ddots & \\ & & & \frac{L^{2}}{z^{2}}\end{array}\right)$.

The area functional to be minimized is given by

$S_{d, k \times R^{d-3}}=L^{d-1} \tilde{H}^{d-3} \int \mathrm{d} \rho \mathrm{d} \theta \frac{\sqrt{\dot{z}^{2}+\rho^{2}\left(1+z^{\prime 2}\right)}}{z^{d-1}}$.

Again one can use the scaling property $z=\rho h(\theta)$, to find the equation of motion:

$h\left(1+h^{2}\right) \ddot{h}+(d-1) \dot{h}^{2}+h^{4}+\mathrm{d} h^{2}+(d-1)=0$.

Equation (3.31) can be integrated to find the following constant along the $\theta$ variation:

$K_{d}=\frac{\left(1+h^{2}\right)^{\frac{(d-1)}{2}}}{h^{(d-1)} \sqrt{h^{2}+h^{2}+1}}=\frac{\left(1+h_{0}^{2}\right)^{\frac{(d-2)}{2}}}{h_{0}^{(d-1)}}$.

Noticing that $h$ is a decreasing function near the boundary, we have from Eq. (3.32)

$\dot{h}=-\frac{\sqrt{1+h^{2}} \sqrt{\left(1+h^{2}\right)^{d-2}-K_{d}^{2} h^{2(d-1)}}}{k_{\mathrm{d}} h^{d-1}}$.

One can find the volume, thus:

$$
\begin{aligned}
V(\gamma)= & L^{d} \tilde{H}^{d-3} \int \rho \mathrm{d} \rho \int \mathrm{d} \theta \int_{\delta}^{z} \frac{\mathrm{d} z}{z^{d}} \\
= & \frac{L^{\mathrm{d}} H^{2} \tilde{H}^{d-3} \Omega}{(d-1) \delta^{d-1}}-\frac{2 L^{d} \tilde{H}^{d-3}}{(d-1)} \\
& \times \int_{\delta / h_{0}}^{H} \frac{\mathrm{d} \rho}{\rho^{d-2}} \int_{h_{0}}^{\delta / \rho} \frac{\mathrm{d} h}{\dot{h} h^{d-1}} .
\end{aligned}
$$

In the limit $h \rightarrow 0$ the integrand in the last term behaves as

$\frac{1}{h^{d-1} \dot{h}} \sim-K_{d}+O\left(h^{2}\right)$.

So we can write

$$
\begin{aligned}
V(\gamma)= & \frac{L^{d} \tilde{H}^{d-3} \Omega H^{2}}{(d-1) \delta^{d-1}} \\
& -\frac{2 L^{d} \tilde{H}^{d-3}}{(d-1)} \int_{\delta / h_{0}}^{H} \frac{\mathrm{d} \rho}{\rho^{d-2}} \\
& \times \int_{h_{0}}^{\delta / \rho} \mathrm{d} h\left(\frac{1}{\dot{h} h^{d-1}}+K_{d}\right)+\frac{2 L^{d} \tilde{H}^{d-3}}{(d-1)} \\
& \times \int_{\delta / h_{0}}^{H} \frac{\mathrm{d} \rho}{\rho^{d-2}} \int_{h_{0}}^{\delta / \rho} \mathrm{d} h K_{d} .
\end{aligned}
$$

We can separate the divergent term as follows:

$$
I_{1}=\int_{\delta / h_{0}}^{H} \frac{\mathrm{d} \rho}{\rho^{d-2}} \int_{h_{0}}^{\delta / \rho} \mathrm{d} h\left(\frac{1}{\dot{h} h^{d-1}}+K_{d}\right) .
$$

Let us denote

$J(h)=\frac{1}{\dot{h} h^{d-1}}+K_{d}$

it is clear from Eq. (3.33) that $J(h) \sim\left(h^{2}\right)$ as $h \rightarrow 0$. We can find the integral $\left(I_{1}\right)$ by integration by parts

$$
\begin{aligned}
I_{1}= & -\frac{1}{(d-3) H^{d-3}} \int_{h_{0}}^{\delta / H} \mathrm{~d} h J(h)-\frac{\delta}{d-3} \\
& \times\left.\int_{\delta / h_{0}}^{H} \frac{\mathrm{d} \rho}{\rho^{d-1}} J(h)\right|_{h=\frac{\delta}{\rho}} \\
= & -\frac{1}{(d-3) H^{d-3}} \int_{h_{0}}^{\delta / H} \mathrm{~d} h J(h)-\frac{\delta}{d-3} I_{2} .
\end{aligned}
$$

Now for finding the divergences of $I_{2}$, we make a change of variable from $\rho$ to $q=\frac{\delta}{\rho}$ and then Taylor expand the terms around $\delta=0$

$$
\begin{aligned}
I_{2}= & -\frac{1}{\delta^{d-2}} \int_{h_{0}}^{\delta / H} \mathrm{~d} q q^{d-3} J(q) \\
= & -\frac{1}{\delta^{d-2}} \\
& \times\left[\int_{h_{0}}^{0} \mathrm{~d} q q^{d-3} J(q)+\frac{\delta}{H}\left(q^{d-3} J(q)\right)_{q=\delta / H}+\cdots\right] \\
= & -\frac{1}{\delta^{d-2}} \int_{h_{0}}^{0} \mathrm{~d} q q^{d-3} J(q)+O\left(\delta^{d}\right) .
\end{aligned}
$$


From Eq. (3.33) $q^{d-3} J(q) \sim q^{d-1}$ for small $q$, hence in the above expression the integral over $q$ is finite. We have also

$I_{1}=\frac{\delta}{d-3} \frac{1}{\delta^{d-2}} \int_{h_{0}}^{0} \mathrm{~d} q q^{d-3} J(q)+$ finite.

So the singular terms of the volume are as follows:

$$
\begin{aligned}
V(\gamma)= & \frac{L^{\mathrm{d}} H^{2} \tilde{H}^{d-3} \Omega}{(d-1) \delta^{d-1}}+\frac{2 K_{d} L^{d} \tilde{H}^{d-3}}{(d-1)} \\
& \times\left[-\frac{h_{0}}{d-3}\left(\frac{h_{0}}{\delta}\right)^{d-3}+\frac{\delta}{d-2}\left(\frac{h_{0}}{\delta}\right)^{(d-2)}\right] \\
& -\frac{2 L^{d} \tilde{H}^{d-3}}{(d-1)(d-3) \delta^{d-3}} \\
& \times \int_{h_{0}}^{0} \mathrm{~d} q q^{d-3} J(q)+\text { finite. }
\end{aligned}
$$

The complexity is finally given by

$\mathcal{C}_{k \times R^{m}}=\frac{V(\gamma)}{8 \pi L G_{N}}$.

\subsection{Conical crease $c_{n} \times R^{m}$}

In this section we consider the special cases of $n=1,2$ and $m=1,2$ in the metric (2.1) which we denote them by cone-crease $c_{n} \times R^{m}$. As in the previous cases the subregion is restricted to the intervals $\theta \in[0, \Omega], \rho \in[0, H]$ and $x_{i} \in[-\tilde{H} / 2, \tilde{H} / 2]$ where $H$ and $\tilde{H}$ indicate IR cut-offs. The extended surface in the bulk is demonstrated by the function $z=z(\rho, \theta)$ with the following induced metric:

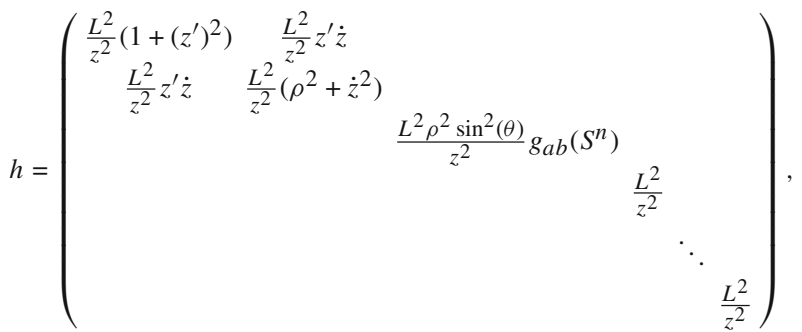

where $g_{[a b]}\left(S^{n}\right)$ is the metric of the sphere $\left(S^{n}\right)$. The surface function to be extremized is the following:

$S_{d, c_{n} \times R^{m}}=L^{d-1} \tilde{H}^{m} \Omega_{n} \int \mathrm{d} \rho \mathrm{d} \theta \frac{\rho^{n} \sin ^{n}(\theta) \sqrt{\dot{z}^{2}+\rho^{2}\left(1+z^{\prime 2}\right)}}{z^{d-1}}$.

The equation of motion for $z(\rho, \theta)$ after imposing the scaling relation $z=\rho h(\theta)$ reads

$$
\begin{aligned}
& h\left(1+h^{2}\right) \ddot{h}+n \cot (\theta) h \dot{h}^{3}+\left(d+n h^{2}-1\right) \dot{h}^{2} \\
& +n \cot (\theta) h\left(1+h^{2}\right) \dot{h} \\
& +(n+1) h^{4}+(d+n) h^{2}+d-1=0 .
\end{aligned}
$$

First consider $n=1$ and $m=1$, i.e. $d=5$. Let us expand $y=\sin (\theta)$ and $\dot{h}$ near the boundary in powers of $h$.

$$
\begin{aligned}
y & =\sin (\Omega)-\frac{1}{6} h^{2} \cos (\Omega) \cot (\Omega) \\
& -\frac{1}{432} h^{4}(19-5 \cos (2 \Omega)) \cot ^{2}(\Omega) \csc (\Omega)+O\left(h^{5}\right), \\
\dot{h}(\theta) & =-\frac{3 \tan (\Omega)}{h}+\frac{1}{3} h(8-\cos (2 \Omega)) \csc (2 \Omega)+f_{0} h^{2} \\
& -\frac{1}{216} h^{3}(435-404 \cos (2 \Omega) \\
& +52 \cos (4 \Omega)) \csc ^{3}(\Omega) \sec (\Omega)+\mathcal{O}\left(h^{4}\right),
\end{aligned}
$$

where $\alpha_{0}$ is fixed by the condition $f\left(h_{0}\right)=0$ at $\mathcal{O}\left(h^{2}\right)$ and vanishes at $\mathcal{O}\left(h^{3}\right)$. Using Eqs. (3.46) and (3.47) the volume functional is as follows:

$$
\begin{aligned}
V(\gamma)= & \frac{L^{5} H^{3} \tilde{H} \Omega_{1}}{12 \delta^{4}}(1-\cos (\Omega)) \\
& -\frac{L^{5} \tilde{H} \Omega_{1}}{4} \int_{\delta / h_{0}}^{H} \frac{d \rho}{\rho^{2}} \int_{h_{0}}^{\delta / \rho} \mathrm{d} h \frac{\sin (\theta)}{\dot{h} h^{4}} \\
= & V_{1}+V_{2} .
\end{aligned}
$$

Near the boundary $h \rightarrow 0$, we have

$$
\begin{aligned}
\frac{\sin (\theta)}{\dot{h} h^{4}} \sim & -\frac{\cos (\Omega)}{3 h^{3}}+\frac{(-13+5 \cos 2 \Omega) \cot (\Omega) \csc (\Omega)}{108 h} \\
& -\frac{1}{9} f_{0} \cos (\Omega) \cot (\Omega) .
\end{aligned}
$$

Now we can use it to make the $h$ integral in holographic complexity finite, i.e.

$$
\begin{aligned}
V_{2}= & -\frac{L^{5} \tilde{H} \Omega_{1}}{4} \int_{\delta / h_{0}}^{H} \frac{\mathrm{d} \rho}{\rho^{2}} \times \int_{h_{0}}^{\delta / \rho} \mathrm{d} h \\
& \times\left[\frac{\sin (\theta)}{\dot{h} h^{4}}+\frac{\cos (\Omega)}{3 h^{3}}-\frac{(-13+5 \cos (2 \Omega)) \cot (\Omega) \csc (\Omega)}{108 h}\right] \\
& -\frac{L^{5} \tilde{H} \Omega_{1}}{4} \int_{\delta / h_{0}}^{H} \frac{\mathrm{d} \rho}{\rho^{2}} \\
& \times \int_{h_{0}}^{\delta / \rho} \mathrm{d} h\left[-\frac{\cos (\Omega)}{3 h^{3}}+\frac{(-13+5 \cos (2 \Omega)) \cot (\Omega) \csc (\Omega)}{108 h}\right] \\
= & I_{1}+I_{2},
\end{aligned}
$$


where

$$
\begin{aligned}
I_{2}= & -\frac{L^{5} \tilde{H} \Omega_{1}}{4}\left[\frac{\cos (\Omega) H}{6 \delta^{2}}\right. \\
& -\frac{1}{\delta}\left(\frac{\cos (\Omega)}{3 h_{0}}+\frac{(-13+5 \cos (2 \Omega)) \cot (\Omega) \csc (\Omega) h_{0}}{108}\right) \\
& \left.-\frac{(-13+5 \cos (2 \Omega)) \cot (\Omega) \csc (\Omega)}{108 H} \log \left(\frac{\delta}{H}\right)\right] .
\end{aligned}
$$

Let us indicate the integrand in $I_{1}$ by $J_{5}(h)$ and integrate it by parts,

$$
\begin{aligned}
I_{1}= & -\frac{L^{5} \tilde{H} \Omega_{1}}{4} \int_{\delta / h_{0}}^{H} \frac{\mathrm{d} \rho}{\rho^{2}} \int_{h_{0}}^{\delta / \rho} \mathrm{d} h J_{5}(h) \\
= & -\frac{L^{5} \tilde{H} \Omega_{1}}{4} \\
& \times\left[-\frac{1}{H} \int_{h_{0}}^{\delta / H} \mathrm{~d} h J_{5}(h)-\left.\delta \int_{\delta / h_{0}}^{H} \frac{\mathrm{d} \rho}{\rho^{3}} J_{5}(h)\right|_{h=\delta / \rho}\right] .
\end{aligned}
$$

Near the boundary $J_{5}(h) \sim \mathcal{O}\left(h^{0}\right)$. We further make the coordinate transformation $q=\delta / \rho$ and Taylor expand the second term of Eq. (3.52) in terms of $\delta$ to find

$$
\begin{aligned}
I_{1}= & -\frac{L^{5} \tilde{H} \Omega_{1}}{4} \\
& \times\left[-\frac{1}{H} \int_{h_{0}}^{\delta / H} \mathrm{~d} h J_{5}(h)+1 / \delta \int_{h_{0}}^{\delta / H} \mathrm{~d} q q J_{5}(q)\right] \\
= & -\frac{L^{5} \tilde{H} \Omega_{1}}{4} \\
& \times\left[-\frac{1}{H} \int_{h_{0}}^{0} \mathrm{~d} h J_{5}(h)+1 / \delta \int_{h_{0}}^{0} \mathrm{~d} q q J_{5}(q)\right. \\
& \left.-\frac{\delta f_{0} \cos (\Omega) \cot (\Omega)}{9 H^{2}}\right]+\mathcal{O}(\delta) .
\end{aligned}
$$

So we have

$$
\begin{aligned}
V(\gamma)= & -\frac{L^{5} \tilde{H} \Omega_{1}}{4}\left[-\frac{H^{3}}{3 \delta^{4}}(\cos (\Omega)-1)+\frac{\cos (\Omega) H}{6 \delta^{2}}\right. \\
& -\frac{1}{\delta}\left(\frac{\cos (\Omega)}{3 h_{0}}+\frac{(-13+5 \cos (2 \Omega)) \cot (\Omega) \csc (\Omega) h_{0}}{108}\right. \\
& \left.-\int_{h_{0}}^{0} \mathrm{~d} q q J_{5}(q)\right) \\
& \left.-\frac{(-13+5 \cos (2 \Omega)) \cot (\Omega) \csc (\Omega)}{108 H} \log \left(\frac{\delta}{H}\right)\right] \\
& + \text { finite. }
\end{aligned}
$$

For $c_{1} \times R^{2}$ the result is as follows:

$$
\begin{aligned}
V(\gamma)= & -\frac{L^{6} \Omega_{1} \tilde{H}^{2}}{5}\left[-\frac{H^{3}(1-\cos (\Omega))}{3 \delta^{5}}+\frac{\cos (\Omega) H}{12 \delta^{3}}\right. \\
& +\frac{1}{\delta^{2}}\left(\frac{-\cos (\Omega)}{8 h_{0}}-\frac{h_{0} \cot (\Omega) \csc (\Omega)(-11+5 \cos (2 \Omega))}{512}\right. \\
& \left.\left.+\frac{1}{2} \int_{h_{0}}^{0} \mathrm{~d} q q^{2} J(q)\right)+\frac{\cot (\Omega) \csc (\Omega)(-11+5 \cos (2 \Omega))}{256 H \delta}\right],
\end{aligned}
$$

where

$$
J(h)=\frac{\sin (\theta)}{h^{5} \dot{h}}+\frac{\cos (\Omega)}{4 h^{4}}-\frac{\cot (\Omega) \csc (\Omega)(-11+5 \cos (2 \Omega))}{256 h^{2}} .
$$

For $c_{2} \times R^{1}$ similar steps lead to

$$
\begin{aligned}
V(\gamma)= & -\frac{\Omega_{2} L^{6} \tilde{H}}{5}\left[-\frac{H^{4}\left(\Omega-\frac{1}{2} \sin (2 \Omega)\right)}{8 \delta^{5}}+\frac{\cos (\Omega) \sin (\Omega) H^{2}}{12 \delta^{3}}\right. \\
& \times \frac{1}{\delta}\left(-\frac{\cos (\Omega) \sin (\Omega)}{4 h_{0}^{2}}-\frac{\cos ^{2}(\Omega) \cot (\Omega)\left(\log \left(h_{0}\right)-1\right)}{16}\right. \\
& \left.\left.+\int_{h_{0}}^{0} \mathrm{~d} q q J(q)\right)+\frac{\cos ^{2}(\Omega) \cot (\Omega)}{16} \frac{1}{\delta} \log \left(\frac{\delta}{H}\right)\right],
\end{aligned}
$$

where

$J(h)=\frac{\sin ^{2}(\theta)}{\dot{h} h^{5}}+\frac{\cos (\Omega) \sin (\Omega)}{2 h^{4}}-\frac{\cos ^{2}(\Omega) \cot (\Omega)}{16 h^{2}}$.

\section{Curved locus singular surfaces}

In this section, we consider several singular embeddings which have a curved locus such as $k \times \Sigma$ and $c_{n} \times \Sigma$, where the locus $\Sigma$ will take the form $S^{m}$ or $S^{m-p} \times R^{p}$.

\subsection{Crease $k \times \Sigma$}

Consider the geometries $k \times S^{2}, k \times S^{3}$ and $k \times R \times S^{2}$. We will see that singularities with even dimensional locus will contribute through a logarithmic term. To begin with, let us consider a $d=5$ CFT on background $R^{3} \times S^{2}$. The action for the six-dimensional dual Einstein gravity reads

$$
I_{6}=\frac{1}{l_{p}^{4}} \int d^{6} x \sqrt{-g}\left[\frac{20}{L^{2}}+R\right] .
$$


We consider the following ansatz for the solution:

$\mathrm{d} s^{2}=\frac{L^{2}}{z^{2}}\left[\mathrm{~d} z^{2}+f_{1}(z)\left(\mathrm{d} t^{2}+\mathrm{d} \rho^{2}+\rho^{2} d \theta^{2}\right)+f_{2}(z) R_{1}^{2} \mathrm{~d} \Omega_{2}^{2}\right]$,

where $\mathrm{d} \Omega_{2}^{2}=d \xi_{0}^{2}+\sin ^{2}\left(\xi_{0}\right) d \xi_{1}^{2}$ represents a two-sphere metric and $f_{1}$ and $f_{2}$ are functions of the radial coordinate. The boundary of this solution is $R^{3} \times S^{2}$ with $R_{1}$ the radius of $S^{2}$; so we can recover the flat boundary results in the limit $R_{1} \rightarrow \infty$. Using the Fefferman-Graham expansion near the boundary to find $f_{1}$ and $f_{2}$ leads to

$$
\begin{aligned}
& f_{1}=1+\frac{z^{2}}{12 R_{1}^{2}}+\frac{17 z^{4}}{576 R_{1}^{4}}-\frac{z^{6}}{324 R_{1}^{6}}+\cdots, \\
& f_{2}=1-\frac{z^{2}}{4 R_{1}^{2}}-\frac{5 z^{4}}{192 R_{1}^{4}}+\frac{z^{6}}{72 R_{1}^{6}}+\cdots
\end{aligned}
$$

The subregion of interest here is $\rho \in[0, H]$ and $\theta \in$ $[-\Omega, \Omega]$ where $H$ is again a IR cut-off. The coordinates are $\left(z, \theta, \xi_{0}, \xi_{1}\right)$ on the minimal surface and $\rho=\rho(z, \theta)$ on the sphere. In the limit $R_{1} \rightarrow \infty$ one may expect from the case of the entanglement entropy that the leading order correction to the holographic subregion complexity would be $\mathcal{O}\left(1 / R_{1}^{2}\right)$, however, we show that in this case there is no new divergent term up to $\mathcal{O}\left(1 / R_{1}^{4}\right)$. We first work out the solution $\rho(z, \theta)$ in this approximation with the following ansatz:

$$
\rho(z, \theta)=\frac{z}{h(\theta)}+\frac{z^{2}}{R_{1}} g_{2}(\theta)+\frac{z^{3}}{R_{1}^{2}} g_{3}(\theta)+\frac{z^{5}}{R_{1}^{4}} g_{5}(\theta)+\mathcal{O}\left(z^{7}\right) .
$$

Using the ansatz (4.5) in the equation of motion of $\rho(z, \theta)$ leads to vanishing of even terms, $g_{2 n}$. In order to separate the logarithmic divergence, we impose $\rho=\rho_{0}(z, \theta)+$ $\rho_{1}(z, \theta) / R_{1}^{2}+\rho_{2}(z, \theta) / R_{1}^{4}$, where $\rho_{0}=z / h(\theta)$, and $\rho_{1}=$ $z^{3} g_{3}(\theta), \rho_{2}=z^{5} g_{5}(\theta)$ are higher corrections in the large $R_{1}$ regime. Now we come back to the metric (4.2) and find the volume holographic complexity to be

$$
\begin{aligned}
V(\gamma) & =L^{5} R_{1}^{2} \Omega_{2} \int \mathrm{d} \rho \mathrm{d} \theta \mathrm{d} z \frac{f_{1} f_{2} \rho}{z^{5}} \\
& =L^{5} R_{1}^{2} \Omega_{2} \int \mathrm{d} \theta \mathrm{d} z \frac{f_{1} f_{2}}{z^{5}} \int_{\rho(z, \theta)}^{H} \mathrm{~d} \rho \rho \\
& =\frac{L^{5} R_{1}^{2} \Omega_{2}}{2}\left(-\int \mathrm{d} \theta \mathrm{d} z \frac{f_{1} f_{2} \rho^{2}}{z^{5}}+H^{2} \int \mathrm{d} \theta \mathrm{d} z \frac{f_{1} f_{2}}{z^{5}}\right) \\
& =V_{1}+V_{2} .
\end{aligned}
$$

Now, we can insert the ansatz $\rho=\rho_{0}+\rho_{1} / R_{1}^{2}+\rho_{2} / R_{1}^{4}$ with $\rho_{0}=z / h(\theta), \rho_{1}=z^{3} g_{3}(\theta)$ and $\rho_{2}=z^{5} g_{5}(\theta)$ and use (4.4) in the integrand to simplify the results,

$$
\begin{aligned}
V_{1}= & L^{5} R_{1}^{2} \Omega_{2}\left[\int_{z_{m}}^{\delta} \frac{\mathrm{d} z}{z^{3}} \int_{h_{0}}^{h_{1} c} \frac{\mathrm{d} h}{\dot{h} h^{2}}-\frac{1}{6 R_{1}^{2}} \int_{z_{m}}^{\delta} \frac{\mathrm{d} z}{z}\right. \\
& \times \int_{h_{0}}^{h_{1} c} \frac{\mathrm{d} h}{\dot{h} h^{2}}+\frac{2}{R_{1}^{2}} \int_{z_{m}}^{\delta} \frac{\mathrm{d} z}{z} \int_{h_{0}}^{h_{1} c} \frac{\mathrm{d} h g_{3}(\theta)}{\dot{h} h} \\
& -\frac{5}{288 R_{1}^{4}} \int_{z_{m}}^{\delta} \mathrm{d} z z \int_{h_{0}}^{h_{1} c} \frac{\mathrm{d} h}{\dot{h} h^{2}}-\frac{1}{3 R_{1}^{4}} \int_{z_{m}}^{\delta} \mathrm{d} z z \\
& \times \int_{h_{0}}^{h_{1} c} \mathrm{~d} h \frac{g_{3}}{\dot{h} h}+\frac{1}{R_{1}^{4}} \int_{z_{m}}^{\delta} \mathrm{d} z z \int_{h_{0}}^{h_{1} c} \mathrm{~d} h \frac{g_{3}^{2}(\theta)}{\dot{h}} \\
& \left.+\frac{2}{R_{1}^{4}} \int_{z_{m}}^{\delta} \mathrm{d} z z \int_{h_{0}}^{h_{1} c} \mathrm{~d} h \frac{g_{5}(\theta)}{\dot{h} h}\right], \\
V_{2}= & L^{5} R_{1}^{2} \Omega_{2} H^{2} \Omega\left(\frac{1}{4 \delta^{4}}-\frac{1}{12 R_{1}^{2} \delta^{2}}\right) \\
& +\frac{5 \Omega L^{5} R_{1}^{2} \Omega_{2} H^{2}}{288 R_{1}^{4}} \log \delta+\text { finite, }
\end{aligned}
$$

where $\delta$ is the UV cut-of. We have also changed the integration limits from $(-\Omega, \Omega)$ to $(0, \Omega)$ and then changed the integration variable in $V_{1}$ to $h(\theta)$. It is instructive to use the following constant of motion:

$K_{5}=\frac{\left(1+h^{2}\right)^{2}}{h^{4} \sqrt{1+h^{2}+\dot{h}^{2}}}$,

which is related to $h(0)$ at the turning point. To find the logarithmic divergent parts it is enough to find the asymptotic behavior of $h$ and $g_{3}$. Solving $g_{3}$ in terms of $h$ in the limit of small $h$ leads to

$$
\begin{aligned}
& g_{3}=\frac{b_{3}}{h^{3}}+\frac{1+88 b_{3}}{56 h}+\frac{4+72 b_{3}}{189} h+\mathcal{O}\left(h^{3}\right), \\
& g_{5}=\frac{9 b_{3}^{2}}{5 h^{5}}+\frac{b_{3}\left(345+15856 b_{3}\right)}{7000 h^{3}}+\mathcal{O}\left(h^{-1}\right),
\end{aligned}
$$

where $b_{3}$ can be fixed by requiring $g_{3}$ to have an extremum at $\theta=0$. We will need to find the series expansion of $h_{1 c}$ in terms of $\delta$ as follows:

$$
\begin{aligned}
h_{1 c}(\delta)= & \left(\frac{1}{H}+\frac{b_{3} H}{R_{1}^{2}}-\frac{b_{3}^{2} H^{3}}{5 R_{1}^{4}}\right) \delta \\
& +\left(\frac{\left(1+88 b_{3}\right)}{56 H R_{1}^{2}}+\frac{\left(1+88 b_{3}\right) H b_{3}}{56 R_{1}^{4}}\right) \delta^{3}+\mathcal{O}\left(\delta^{5}\right)
\end{aligned}
$$

where $h_{1 c}=h(\Omega-\epsilon)$. The result is obtained for the leading corrections in $R_{1}$ at any order of $\delta$. Now we look at (4.7) to analyze the divergent terms in the asymptotic limit 
665 Page 12 of 17

Eur. Phys. J. C (2017) 77:665

$$
\frac{1}{\dot{h} h^{2}} \sim-K_{5} h^{2}+2 K_{5} h^{4}+\mathcal{O}\left(h^{6}\right)
$$

and

$$
\begin{aligned}
\frac{g_{3}}{\dot{h}} & \sim-K_{5} b_{3}-\frac{K_{5}\left(1-24 b_{3}\right) h^{2}}{56}+\mathcal{O}\left(h^{4}\right), \\
\frac{g_{3}^{2}}{\dot{h}} & \sim-\frac{K_{5} b_{3}^{2}}{h^{2}}-\frac{k_{5}\left(b_{3}+32 b_{3}^{2}\right)}{28}+\mathcal{O}\left(h^{2}\right), \\
\frac{g_{5}}{\dot{h} h} & \sim-\frac{9 K_{5} b_{3}^{2}}{5 h^{2}}-\frac{b_{3}\left(345-9344 b_{3}\right) K_{5}}{7000}+\mathcal{O}\left(h^{2}\right) .
\end{aligned}
$$

We organize different terms of the integrand in the following form:

$$
\begin{aligned}
I_{1}= & \int_{z m}^{\delta} \frac{\mathrm{d} z}{z^{3}} \int_{h_{0}}^{h_{1} c} \frac{\mathrm{d} h}{\dot{h} h^{2}}, \\
I_{2}= & \int_{z_{m}}^{\delta} \frac{\mathrm{d} z}{z} \int_{h_{0}}^{h_{1} c} \frac{\mathrm{d} h}{\dot{h} h^{2}}, \\
I_{3}= & \int_{z_{m}}^{\delta} \frac{\mathrm{d} z}{z} \int_{h_{0}}^{h_{1} c} \frac{\mathrm{d} h g_{3}}{\dot{h} h}=\int_{z_{m}}^{\delta} \frac{\mathrm{d} z}{z} \int_{h_{0}}^{h_{1} c} \\
& \times \mathrm{d} h\left(\frac{g_{3}}{\dot{h} h}+K_{5} b_{3}\right)-K_{5} b_{3} \int_{z_{m}}^{\delta} \frac{\mathrm{d} z}{z}\left(h_{1 c}-h_{0}\right) \\
= & I_{1}^{\prime}+I_{2}^{\prime}, \\
I_{1}^{\prime \prime}= & \int_{z_{m}}^{\delta} z \mathrm{~d} z \int_{h_{0}}^{h_{1} c} \frac{\mathrm{d} h}{\dot{h} h^{2}}, \\
I_{2}^{\prime \prime}= & \int_{z_{m}}^{\delta} z \mathrm{~d} z \int_{h_{0}}^{h_{1} c} \mathrm{~d} h \frac{g_{3}}{\dot{h} h^{2}}, \\
I_{3}^{\prime \prime}= & \int_{z_{m}}^{\delta} z \mathrm{~d} z \int_{h_{0}}^{h_{1} c} \mathrm{~d} h\left(\frac{g_{3}^{2}}{\dot{h}}+\frac{K_{5} b_{3}^{2}}{h^{2}}\right) \\
& -\int_{z_{m}}^{\delta} z \mathrm{~d} z \int_{h_{0}}^{h_{1} c} \mathrm{~d} h \frac{K_{5} b_{3}^{2}}{h^{2}} \\
= & I_{1}^{\prime \prime \prime}+I_{2}^{\prime \prime \prime}, \\
I_{4}^{\prime \prime}= & \int_{z_{m}}^{\delta} z \mathrm{~d} z \int_{h_{0}}^{h_{1} c} \mathrm{~d} h\left(\frac{g_{5}}{\dot{h} h}+\frac{9 K_{5} b_{3}^{2}}{5 h^{2}}\right) \\
& -\int_{z_{m}}^{\delta} z \mathrm{~d} z \int_{h_{0}}^{h_{1} c} \mathrm{~d} h \frac{9 K_{5} b_{3}^{2}}{5 h^{2}} \\
= & I_{3}^{\prime \prime \prime}+I_{4}^{\prime \prime \prime} .
\end{aligned}
$$

Now we differentiate each of them with respect to the UV cutoff and look for $1 / \delta$ divergent terms. One can easily find

$$
\begin{aligned}
\frac{\mathrm{d} I_{1}}{d \delta} & =\frac{1}{\delta^{3}} \int_{h_{0}}^{h_{1 c}} \frac{\mathrm{d} h}{\dot{h} h^{2}} \\
& =\frac{1}{\delta^{3}} \int_{h_{0}}^{0} \frac{\mathrm{d} h}{\dot{h} h^{2}}+\frac{1}{\delta^{2}} \frac{d h_{1 c}}{d \delta}\left[\frac{1}{\dot{h} h^{2}}\right]_{h=h_{1 c}}+\cdots \\
& =\frac{1}{\delta^{3}} \int_{h_{0}}^{0} \frac{\mathrm{d} h}{\dot{h} h^{2}}+\mathcal{O}\left(\delta^{0}\right)
\end{aligned}
$$

$$
\begin{aligned}
\frac{\mathrm{d} I_{2}}{d \delta} & =\frac{1}{\delta} \int_{h_{0}}^{h_{1 c}} \frac{\mathrm{d} h}{\dot{h} h^{2}} \\
& =\frac{1}{\delta} \int_{h_{0}}^{0} \frac{\mathrm{d} h}{\dot{h} h^{2}}+\frac{d h_{1 c}}{d \delta}\left[\frac{1}{\dot{h} h^{2}}\right]_{h=h_{1 c}}+\cdots \\
& =\frac{1}{\delta} \int_{h_{0}}^{0} \frac{\mathrm{d} h}{\dot{h} h^{2}}+\mathcal{O}\left(\delta^{2}\right) \\
\frac{\mathrm{d} I_{1}^{\prime}}{d \delta} & =\frac{1}{\delta} \int_{h_{0}}^{h_{1} c} \mathrm{~d} h\left[\frac{g_{3}}{\dot{h} h}+K_{5} b_{3}\right] \\
= & \frac{1}{\delta} \int_{h_{0}}^{0} \mathrm{~d} h\left(\frac{g_{3}}{\dot{h} h}+K_{5} b_{3}\right)+\frac{d h_{1 c}}{d \delta}\left[\frac{g_{3}}{\dot{h} h}+K_{5} b_{3}\right]_{h=h_{1 c}}+\ldots \\
= & \frac{1}{\delta} \int_{h_{0}}^{0} \mathrm{~d} h\left(\frac{g_{3}}{\dot{h} h}+K_{5} b_{3}\right)+\mathcal{O}\left(\delta^{2}\right) \\
\frac{\mathrm{d} I_{2}^{\prime}}{d \delta}= & -\frac{K_{5} b_{3}}{\delta}\left(h_{1 c}-h_{0}\right)=\frac{h_{0} K_{5} b_{3}}{\delta} \\
& -\frac{K_{5} b_{3}}{H}\left(1+b_{3} H^{2} / R_{1}^{2}-b_{3}^{2} H^{4} / 5 R_{1}^{4}\right)+\mathcal{O}\left(\delta^{2}\right)
\end{aligned}
$$

$\frac{\mathrm{d} I_{1}^{\prime \prime}}{d \delta}=\delta \int_{h_{0}}^{h_{1 c}} \frac{\mathrm{d} h}{\dot{h} h^{2}}$$$
=\delta \int_{h_{0}}^{0} \frac{\mathrm{d} h}{\dot{h} h^{2}}+\delta^{2} \frac{d h_{1 c}}{d \delta}\left[\frac{1}{\dot{h} h^{2}}\right]_{h=h_{1 c}}+\cdots
$$$$
=\delta \int_{h_{0}}^{0} \frac{\mathrm{d} h}{\dot{h} h^{2}}+\mathcal{O}\left(\delta^{4}\right),
$$$$
\begin{aligned}
\frac{\mathrm{d} I_{2}^{\prime \prime}}{d \delta} & =\delta \int_{h_{0}}^{h_{1 c}} \mathrm{~d} h \frac{g_{3}}{\dot{h} h} \\
& =\delta \int_{h_{0}}^{0} \mathrm{~d} h \frac{g_{3}}{\dot{h} h^{2}}+\delta^{2} \frac{d h_{1 c}}{d \delta} \\
& =\delta \int_{h_{0}}^{0} \frac{\mathrm{d} h}{\dot{h} h^{2}}+\mathcal{O}\left(\delta^{2}\right),
\end{aligned}
$$$$
\frac{\mathrm{d} I_{1}^{\prime \prime \prime}}{d \delta}=\delta \int_{h_{0}}^{h_{1 c}} \mathrm{~d} h\left(\frac{g_{3}^{2}}{\grave{h}}+\frac{K_{5} b_{3}^{2}}{h^{2}}\right)
$$$$
=\delta \int_{h_{0}}^{0} \mathrm{~d} h\left(\frac{g_{3}^{2}}{\grave{h}}+\frac{K_{5} b_{3}^{2}}{h^{2}}\right)+\delta^{2} \frac{d h_{1 c}}{d \delta}\left[\frac{g_{3}^{2}}{\grave{h}}+\frac{K_{5} b_{3}^{2}}{h^{2}}\right]_{h=h_{1 c}}+\cdots
$$$$
=\delta \int_{h_{0}}^{0} \mathrm{~d} h\left(\frac{g_{3}^{2}}{\grave{h}}+\frac{K_{5} b_{3}^{2}}{h^{2}}\right)+\mathcal{O}\left(\delta^{2}\right) .
$$

$\frac{\mathrm{d} I_{2}^{\prime \prime \prime}}{d \delta}=-\delta \int_{h_{0}}^{h_{1 c}} \frac{K_{5} b_{3}^{2}}{h^{2}}$

$$
\begin{aligned}
& =\delta K_{5} b_{3}^{2}\left(\frac{1}{h_{1 c}}-\frac{1}{h_{0}}\right) \\
& =-\frac{\delta K_{5} b_{3}^{2}}{h_{0}}+K_{5} b_{3}^{2} H\left[1-\frac{b_{3} H^{2}}{R_{1}^{2}}+\frac{b_{3}^{2} H^{4}}{5 R_{1}^{4}}\right]+\mathcal{O}\left(\delta^{2}\right),
\end{aligned}
$$

$$
\begin{aligned}
\frac{\mathrm{d} I_{3}^{\prime \prime \prime}}{d \delta} & =\delta \int_{h_{0}}^{h_{1 c}} \mathrm{~d} h\left(\frac{g_{5}}{\dot{h} h}+\frac{9 K_{5} b_{3}^{2}}{5 h^{2}}\right) \\
& =\delta \int_{h_{0}}^{0} \mathrm{~d} h\left(\frac{g_{5}}{\grave{h} h}+\frac{9 K_{5} b_{3}^{2}}{5 h^{2}}\right)+\delta^{2} \frac{d h_{1 c}}{d \delta}\left[\frac{g_{5}}{\grave{h} h}+\frac{9 K_{5} b_{3}^{2}}{5 h^{2}}\right]_{h=h_{1 c}}+\cdots \\
& =\delta \int_{h_{0}}^{0} \mathrm{~d} h\left(\frac{g_{5}}{\grave{h} h}+\frac{9 K_{5} b_{3}^{2}}{5 h^{2}}\right)+\mathcal{O}\left(\delta^{2}\right)
\end{aligned}
$$

Springer 


$$
\begin{aligned}
\frac{\mathrm{d} I_{4}^{\prime \prime \prime}}{d \delta} & =-\delta \int_{h_{0}}^{h_{1 c}} \frac{9 K_{5} b_{3}^{2}}{5 h^{2}} \\
& =\delta \frac{9 K_{5} b_{3}^{2}}{5}\left(\frac{1}{h_{1 c}}-\frac{1}{h_{0}}\right) \\
& =-\delta \frac{9 K_{5} b_{3}^{2}}{5 h_{0}}+\frac{9 K_{5} b_{3}^{2} H}{5}\left[1-\frac{b_{3} H^{2}}{R_{1}^{2}}+\frac{b_{3}^{2} H^{4}}{5 R_{1}^{4}}\right]+\mathcal{O}\left(\delta^{2}\right) .
\end{aligned}
$$

So from (4.17)-(4.27) we can find the logarithmic divergences in the holographic complexity for the $k \times S^{2}$ geometry as follows:

$$
\begin{aligned}
\mathcal{C}_{k \times S^{2}}^{\log }= & \frac{L^{5} R_{1}^{2} \Omega_{2}}{8 \pi L G}\left[-\frac{1}{6 R_{1}^{2}} \int_{h_{0}}^{0} \frac{\mathrm{d} h}{\dot{h} h^{2}}+\frac{2}{R_{1}^{2}} \int_{h_{0}}^{0} \mathrm{~d} h\right. \\
& \left.\times\left(\frac{g_{3}}{\dot{h} h}+K_{5} b_{3}\right)+\frac{2 h_{0} K_{5} b_{3}}{R_{1}^{2}}+\frac{5 \Omega L^{5} \Omega_{2} H^{2}}{288 R_{1}^{2}}\right] \\
& \times \log (\delta) .
\end{aligned}
$$

Note that in this case no new divergent term appears due to the singular surface. All new $\log \delta$ terms are suppressed with a factor of $\delta^{\alpha}$ where $\alpha \geq 1$.

\section{Subregion $k \times S^{3}$}

Now we want to find the holographic subregion complexity for the $k \times S^{3}$ geometry in a CFT on $R^{3} \times S^{3}$. We will show that in this case the singularity gives no logarithmic contribution to the subregion complexity. Consider the following metric:

$\mathrm{d} s^{2}=\frac{L^{2}}{z^{2}}\left[\mathrm{~d} z^{2}+f_{1}(z)\left(\mathrm{d} t^{2}+\mathrm{d} \rho^{2}+\rho^{2} \mathrm{~d} \theta^{2}\right)+f_{2}(z) R_{1}^{2} \mathrm{~d} \Omega_{3}^{2}\right]$,

where $\mathrm{d} \Omega_{3}^{2}=d \xi_{0}^{2}+\sin ^{2}\left(\xi_{0}\right) \mathrm{d} \xi_{1}^{2}+\sin ^{2}\left(\xi_{0}\right) \sin ^{2}\left(\xi_{1}\right) d \xi_{2}^{2}$ is the unit $S^{3}$ and we find $f_{1}$ and $f_{2}$ to be

$$
\begin{aligned}
& f_{1}=1+\frac{3 z^{2}}{20 R_{1}^{2}}+\frac{69 z^{4}}{1600 R_{1}^{4}}+\frac{z^{6}}{R_{1}^{6}}\left(\frac{33}{8000}-\frac{1}{200} \log z\right)+\mathcal{O}\left(z^{8}\right) \\
& f_{2}=1-\frac{7 z^{2}}{20 R_{1}^{2}}-\frac{11 z^{4}}{1600 R_{1}^{4}}+\frac{z^{6}}{R_{1}^{6}}\left(\frac{67}{8000}+\frac{1}{200} \log z\right)+\mathcal{O}\left(z^{8}\right) .
\end{aligned}
$$

Similar to the previous case the induced coordinates on the RT surface are $\left(z, \theta, \xi_{0}, \xi_{1}, \xi_{2}\right)$ and $\rho=\rho(z, \theta)$. Using the equation of motion for $h$ we can find the following constant of motion:

$$
K_{6}=\frac{\left(1+h^{2}\right)^{5 / 2}}{h^{5} \sqrt{1+h^{2}+\dot{h}^{2}}},
$$

which can be fixed in terms of the boundary data. Using the metric (4.35) we find the holographic complexity as

$$
\begin{aligned}
V(\gamma)= & L^{6} R_{1}^{3} \Omega_{3} \int \mathrm{d} \rho \mathrm{d} \theta \mathrm{d} z \frac{f_{1} f_{2}^{3 / 2} \rho}{z^{6}} \\
= & L^{6} R_{1}^{3} \Omega_{3} \int \mathrm{d} \theta \mathrm{d} z \frac{f_{1} f_{2}^{3 / 2}}{z^{6}} \int_{\rho(z, \theta)}^{H} \mathrm{~d} \rho \rho=\frac{L^{6} R_{1}^{3} \Omega_{3}}{2} \\
& \times\left(-\int \mathrm{d} \theta \mathrm{d} z \frac{f_{1} f_{2}^{3 / 2} \rho^{2}}{z^{6}}+H^{2} \int \mathrm{d} \theta \mathrm{d} z \frac{f_{1} f_{2}^{3 / 2}}{z^{6}}\right) \\
= & V_{1}+V_{2} .
\end{aligned}
$$

Inserting the ansatz $\rho=\rho_{0}+\rho_{1} / R_{1}^{2}, \rho_{0}=z / h(\theta)$ and $\rho_{1}=z^{3} g_{3}(\theta)$ and using the expansions (4.36) in the integrand simplify the result to

$$
\begin{aligned}
V_{1}= & L^{6} R_{1}^{3} \Omega_{3} \int_{z_{m}}^{\delta} \frac{\mathrm{d} z}{z^{2}} \int_{h_{0}}^{h_{1} c} \frac{\mathrm{d} h}{\dot{h} h^{2}} \\
& \times\left(\frac{1}{2 z^{2}}-\frac{9}{20 R_{1}^{2}}+\frac{1}{R_{1}^{2}} h g_{3}(\theta)\right)
\end{aligned}
$$

and

$V_{2}=L^{6} R_{1}^{3} \Omega_{3} H^{2} \Omega\left(\frac{1}{10 \delta^{5}}-\frac{9}{60 R_{1}^{2} \delta^{3}}\right)+$ finite

where $\delta$ is the UV cut-of, such that $\rho(z, \Omega-\epsilon)=H$ and $z_{m}$ is defined such that $\rho\left(z_{m}, 0\right)=H$. We have also changed the integration limits from $(-\Omega, \Omega)$ to $(0, \Omega)$ and then changed the integration variable in $V_{1}$ to $h(\theta)$.

Similar to what we have done in the previous sections in detail, one can work out the logarithmic divergence in this case. Here we step the details and report to the final result

$$
\begin{aligned}
\mathcal{C}_{k \times S^{3}}= & \frac{L^{6} R_{1}^{3} \Omega_{3}}{8 \pi L G_{N}}\left[\frac{\Omega H^{2}}{10 \delta^{5}}-\frac{9 \Omega H^{2}}{60 R_{1}^{2} \delta^{3}}-\frac{1}{6 \delta^{3}} \int_{h_{0}}^{0} \frac{\mathrm{d} h}{\dot{h} h^{2}}+\frac{9}{20 R_{1}^{2} \delta}\right. \\
& \left.\times \int_{h_{0}}^{0} \frac{\mathrm{d} h}{\dot{h} h^{2}}-\frac{1}{R_{1}^{2} \delta} \int_{h_{0}}^{0} \mathrm{~d} h \frac{g_{3}}{\dot{h} h}\right]
\end{aligned}
$$

In this case no new divergent term appears due to the singular surface and all new $\log \delta$ terms are suppressed with a factor of $\delta^{\alpha}$ where $\alpha \geq 1$.

Subregion $k \times R^{1} \times S^{2}$

In the following we give another example showing that an odd dimensional locus does not contribute to logarithmic singularities, although it has non-zero curvature. We consider a CFT defined on $R^{4} \times S^{2}$. The bulk metric is given by 


$$
\begin{aligned}
\mathrm{d} s^{2}= & \frac{L^{2}}{z^{2}}\left[\mathrm{~d} z^{2}+f_{1}(z)\left(\mathrm{d} t^{2}+\mathrm{d} \rho^{2}+\rho^{2} \mathrm{~d} \theta^{2}+\mathrm{d} x^{2}\right)\right. \\
& \left.+f_{2}(z) R_{1}^{2} \mathrm{~d} \Omega_{2}^{2}\right]
\end{aligned}
$$

where $\mathrm{d} \Omega_{2}$ is the line element over $S^{2}$, and $f_{1}$ and $f_{2}$ have the following expansions:

$$
\begin{aligned}
& f_{1}=1+\frac{z^{2}}{20 R_{1}^{2}}+\frac{z^{4}}{100 R_{1}^{4}}+\frac{z^{6}}{R_{1}^{6}}\left(\frac{1}{1200}-\frac{1}{400} \log z\right)+\mathcal{O}\left(z^{8}\right), \\
& f_{2}=1-\frac{z^{2}}{5 R_{1}^{2}}-\frac{7 z^{4}}{800 R_{1}^{4}}+\frac{z^{6}}{R_{1}^{6}}\left(\frac{7}{4800}+\frac{1}{200} \log z\right)+\mathcal{O}\left(z^{8}\right) .
\end{aligned}
$$

The subregion $k \times R^{1} \times S^{2}$ is defined by $\theta \in[-\Omega, \Omega], x \in$ $[-\infty, \infty]$ and $\rho \in[0, \infty]$. we put IR cut-offs on the $x$ and $\rho$ directions such that $x \in[-\tilde{H} / 2, \tilde{H} / 2]$ and $\rho \in\left[\rho_{m}, H\right]$, where $\rho_{m}$ is given in terms of $\delta$. Similar to the previous cases $\left(z, \theta, x, \xi_{0}, \xi_{1}\right)$ are the coordinates on the RT surface with $\rho=\rho(z, \theta)$. The equation of motion for $h$ gives the following constant of motion:

$K_{6}=\frac{\left(1+h^{2}\right)^{5 / 2}}{h^{5} \sqrt{1+h^{2}+\dot{h}^{2}}}$.

Returning to the metric (4.42) we find the holographic complexity as

$$
\begin{aligned}
V(\gamma)= & L^{6} R_{1}^{2} \tilde{H} \Omega_{2} \int \mathrm{d} \rho \mathrm{d} \theta \mathrm{d} z \frac{f_{2} f_{1}^{3 / 2} \rho}{z^{6}} \\
= & L^{6} R_{1}^{2} \tilde{H} \Omega_{2} \int \mathrm{d} \theta \mathrm{d} z \frac{f_{2} f_{1}^{3 / 2}}{z^{6}} \int_{\rho(z, \theta)}^{H} \mathrm{~d} \rho \rho \\
= & \frac{L^{6} R_{1}^{2} \tilde{H} \Omega_{2}}{2} \\
& \times\left(-\int \mathrm{d} \theta \mathrm{d} z \frac{f_{2} f_{1}^{3 / 2} \rho^{2}}{z^{6}}+H^{2} \int \mathrm{d} \theta \mathrm{d} z \frac{f_{2} f_{1}^{3 / 2}}{z^{6}}\right) \\
= & V_{1}+V_{2} .
\end{aligned}
$$

We then insert the ansatz $\rho=\rho_{0}+\rho_{1} / R_{1}^{2}, \rho_{0}=z / h(\theta)$ and $\rho_{1}=z^{3} g_{3}(\theta)$ and use (4.43) in the integrand to simplify the expressions as follows:

$$
\begin{aligned}
V_{1}= & -L^{6} R_{1}^{2} \tilde{H} \Omega_{2} \int_{\delta}^{z_{m}} \frac{\mathrm{d} z}{z^{2}} \int_{h_{0}}^{h_{1} c} \frac{\mathrm{d} h}{\dot{h} h^{2}} \\
& \times\left(\frac{1}{z^{2}}-\frac{1}{8 R_{1}^{2}}+\frac{2}{R_{1}^{2}} h g_{3}(\theta)\right), \\
V_{2}= & L^{6} R_{1}^{2} \Omega_{2} H^{2} \tilde{H} \Omega\left(\frac{1}{5 \delta^{5}}-\frac{1}{24 R_{1}^{2} \delta^{3}}\right)+\text { finite. }
\end{aligned}
$$

Again we step over the details of the rest of this calculation and find

$$
\begin{aligned}
\mathcal{C}_{k \times R^{1} \times S^{1}}= & \frac{L^{6} R_{1}^{2} \tilde{H} \Omega_{2}}{8 \pi L G}\left(\frac{\Omega H^{2}}{5 \delta^{5}}-\frac{\Omega H^{2}}{24 R_{1}^{2} \delta^{3}}\right. \\
& \left.-\frac{1}{3 \delta^{3}} \int_{h_{0}}^{0} \frac{\mathrm{d} h}{\grave{h} h^{2}}+\frac{1}{8 R_{1}^{2} \delta} \int_{h_{0}}^{0} \frac{\mathrm{d} h}{\grave{h} h^{2}}-\frac{2}{R_{1}^{2} \delta} \int_{h_{0}}^{0} \mathrm{~d} h \frac{g_{3}}{\dot{h} h}\right) .
\end{aligned}
$$

As in the case of $k \times S^{2}$ and $k \times S^{3}$ new logarithmic divergent terms in this case are also suppressed by a factor of $\delta^{\alpha}$ with a positive power.

\subsection{Conical crease $c_{n} \times \Sigma$}

In this section, we will calculate the holographic complexity for subregions with conical singularities of the form $c_{n} \times S^{m}$.

\section{Subregion $c_{1} \times S^{1}$}

To begin with, we consider the simplest case with $m=1$. In this case, the background geometry for CFT is $R^{4} \times S^{1}$. The dual bulk geometry is then given by

$$
\begin{aligned}
\mathrm{d} s^{2}= & \frac{L^{2}}{z^{2}}\left[\mathrm{~d} z^{2}+f_{1}(z)\left(\mathrm{d} t^{2}+\mathrm{d} \rho^{2}+\rho^{2} \mathrm{~d} \theta^{2}\right.\right. \\
& \left.\left.+\rho^{2} \sin ^{2}(\theta) d \phi^{2}\right)+f_{2}(z) R_{1}^{2} d \xi_{0}^{2}\right],
\end{aligned}
$$

where $f_{1}=1+O\left(1 / R_{1}^{6}\right)$ and $f_{2}=1+O\left(1 / R_{1}^{6}\right)$. The singular subregion of our interest is defined as $\theta \in[0, \Omega], \xi_{0} \in$ $[0,2 \pi], \phi \in[0,2 \pi]$ and $\rho \in[0, H]$.

One can find that $g_{3}=0$ is the exact solution for this case and since the equation of motion for $h$ is the same as $c_{1} \times R^{1}$ case, the holographic subregion complexity might become the same. Returning to the metric (4.49) gives the holographic complexity:

$$
\begin{aligned}
V(\gamma)= & L^{5} R_{1} 4 \pi^{2} \int \mathrm{d} \rho \mathrm{d} \theta \mathrm{d} z \frac{f_{2}^{1 / 2} f_{1}^{3 / 2} \rho^{2} \sin (\theta)}{z^{5}} \\
= & L^{5} R_{1} 4 \pi^{2} \int \mathrm{d} \theta \mathrm{d} z \frac{f_{2}^{1 / 2} f_{1}^{3 / 2} \sin (\theta)}{z^{5}} \int_{\rho(z, \theta)}^{H} \mathrm{~d} \rho \rho^{2} \\
= & \frac{L^{5} R_{1} 4 \pi^{2}}{3} \\
& \times\left(-\int \mathrm{d} \theta \mathrm{d} z \frac{f_{2}^{1 / 2} f_{1}^{3 / 2} \rho^{3}}{z^{5}}+H^{3} \int \mathrm{d} \theta \mathrm{d} z \frac{f_{2}^{1 / 2} f_{1}^{3 / 2}}{z^{5}}\right) .
\end{aligned}
$$

A similar analysis to the previous cases leads to the following divergence structure for the holographic subregion complexity for this case: 


$$
\begin{aligned}
\mathcal{C}_{c_{1} \times S^{1}}= & \frac{L^{4} R_{1} \pi}{6 G_{N}}\left[\frac{1}{4 \delta^{4}} H^{3}(1-\cos (\Omega))-\frac{\cos (\Omega) H}{6 \delta^{2}}\right. \\
& +\frac{1}{\delta}\left(\frac{\cos (\Omega)}{3 h_{0}}+\frac{(-13+5 \cos (2 \Omega)) \cot (\Omega) \csc (\Omega) h_{0}}{108}\right. \\
& -\frac{\cos (\Omega) \cot (\Omega) f_{0} h_{0}^{2}}{18} \\
& -\int_{h_{0}}^{0} \mathrm{~d} h\left(\frac{\sin (\theta)}{\dot{h} h^{3}}+\frac{\cos (\Omega)}{3 h^{2}}\right. \\
& \left.\left.-\frac{(-13+5 \cos (2 \Omega)) \cot (\Omega) \csc (\Omega)}{108}+\frac{\cos (\Omega) \cot (\Omega) f_{0} h}{9}\right)\right) \\
& \left.+\frac{(-13+5 \cos (2 \Omega)) \cot (\Omega) \csc (\Omega)}{108 H} \log (\delta)\right]+ \text { finite. }
\end{aligned}
$$

Subregion $c_{1} \times S^{2}$

Next we consider the singular subregion $c_{1} \times S^{2}$ in a CFT defined on $R^{4} \times S^{2}$. The bulk metric is given by

$$
\begin{aligned}
\mathrm{d} s^{2} & =\frac{L^{2}}{z^{2}}\left[\mathrm{~d} z^{2}+f_{1}(z)\left(\mathrm{d} t^{2}+\mathrm{d} \rho^{2}+\rho^{2} \mathrm{~d} \theta^{2}\right.\right. \\
& \left.\left.+\rho^{2} \sin (\theta)^{2} d \phi^{2}\right)+f_{2}(z) R_{1}^{2} d \Omega_{2}^{2}\right],
\end{aligned}
$$

where $\mathrm{d} \Omega_{2}$ is the line element over $S^{2}$, and $f_{1}$ and $f_{2}$ have the following expansions:

$$
\begin{aligned}
& f_{1}=1+\frac{z^{2}}{20 R_{1}^{2}}+\frac{z^{4}}{100 R_{1}^{4}}+\frac{z^{6}}{R_{1}^{6}}\left(\frac{1}{1200}-\frac{1}{400} \log z\right)+\mathcal{O}\left(z^{8}\right), \\
& f_{2}=1-\frac{z^{2}}{5 R_{1}^{2}}-\frac{7 z^{4}}{800 R_{1}^{4}}+\frac{z^{6}}{R_{1}^{6}}\left(\frac{7}{4800}+\frac{1}{200} \log z\right)+\mathcal{O}\left(z^{8}\right) .
\end{aligned}
$$

Using the metric (4.51) we find the holographic complexity as

$$
\begin{aligned}
V(\gamma)= & 2 \pi L^{6} R_{1}^{2} \Omega_{2} \int \mathrm{d} \rho \mathrm{d} \theta \mathrm{d} z \frac{f_{2} f_{1}^{3 / 2} \rho^{2} \sin (\theta)}{z^{6}} \\
= & 2 \pi L^{6} R_{1}^{2} \Omega_{2} \int \mathrm{d} \theta \mathrm{d} z \frac{f_{2} f_{1}^{3 / 2} \sin (\theta)}{z^{6}} \int_{\rho(z, \theta)}^{H} \mathrm{~d} \rho \rho^{2} \\
= & \frac{2 \pi L^{6} R_{1}^{2} \Omega_{2}}{3} \\
& \times\left(-\int \mathrm{d} \theta \mathrm{d} z \frac{f_{2} f_{1}^{3 / 2} \rho^{3}}{z^{6}}+H^{3} \int \mathrm{d} \theta \mathrm{d} z \frac{f_{2} f_{1}^{3 / 2}}{z^{6}}\right) .
\end{aligned}
$$

A similar analysis to the previous sections leads to

$$
\begin{aligned}
\mathcal{C}_{c_{1} \times S^{2}}^{\log }= & \frac{L^{5} R_{1}^{2} \Omega_{2}}{12 G_{N}}\left[\frac { 3 } { R _ { 1 } ^ { 2 } } \left(\int _ { h _ { 0 } } ^ { 0 } \mathrm { d } h \left[\frac{\sin (\theta) g_{3}}{\dot{h} h^{2}}-\frac{\cos (\Omega)}{80 h^{2}}\right.\right.\right. \\
& +\frac{\cos (\Omega)\left(1+\csc ^{2}(\Omega)\right) \log (h)}{384}
\end{aligned}
$$

$$
\begin{aligned}
& \left.+\frac{\cos (\Omega)\left(-3+27 \cot ^{2}(\Omega)-45 \csc ^{2}(\Omega)+3840 b_{3}\right)}{15360}\right] \\
& +\frac{\cos (\Omega)}{80 h_{0}} \\
& +\frac{\cos (\Omega)\left(1+\csc ^{2}(\Omega)\right)}{384}\left(h_{0} \log \left(h_{0}\right)-h_{0}\right) \\
& \left.+\frac{\cos (\Omega)\left(-3+27 \cot ^{2}(\Omega)-45 \csc ^{2}(\Omega)+3840 b_{3}\right) h_{0}}{15360}\right) \\
& -\frac{1}{8 R_{1}^{2}}\left(\int _ { h _ { 0 } } ^ { 0 } \mathrm { d } h \left[\frac{\sin (\theta)}{\dot{h} h^{3}}+\frac{\cos (\Omega)}{4 h^{2}}\right.\right. \\
& \left.-\frac{\cos (\Omega) \csc ^{2}(\Omega)(-11+5 \cos (2 \Omega))}{256}\right] \\
& \left.\left.-\frac{\cos (\Omega)}{4 h_{0}}-\frac{\cos (\Omega) \csc ^{2}(\Omega) h_{0}(-11+\cos (2 \Omega))}{256}\right)\right] \log (\delta) .
\end{aligned}
$$

\section{Discussions}

In this paper we studied the divergence structure of holographic subregion complexity for various singular surfaces. We showed that there are new divergences due to singularities in the subregion. More specifically we have shown that for a kink in a (2+1)-dimensional field theory and also cones $c_{n}$ in even dimensional field theories a new universal $\log \delta$ term appears. In odd dimensional field theories the singularity of a cone $c_{n}$ gives rise to a $\log ^{2} \delta$ divergent term. We also showed that surprisingly crease singularities of any type do not give rise to any universal term or even any new divergent term. For generalized conical singularities the situation is completely different. There are examples for which new power law divergences appear but there is no new universal term due to the singularity. We found also an example, i.e. $c_{1} \times S^{2}$, with a curved locus that has a new universal term. Another type of conical singularity has $\frac{1}{\delta} \log \delta$ and $\frac{1}{\delta^{2}} \log \delta$ divergent terms for even and odd dual field theories, respectively. The latter family is very similar to what has been recently found using the 'complexity = action' proposal on the Wheeler-DeWitt patch which also possesses corners. We have summarized all of these results in a table in Sect. 2.

There are several directions to follow in future work. Regarding the divergence structure of subregion complexity, the most important question is whether one can define any monotonic function from the universal terms which leads to a kind of 'c-function' in higher odd-dimensional dual field theories.

Another interesting open question is how to generalize complexity proposals beyond Einstein gravity. Recently there have been some proposals trying to address this question (see e.g. [39]).

A natural question as regards this work is how to study the role of singularities of subregions in the "complexity = action' proposal. Recently some progress have been made in 
[11] for spherical subregions. The authors have proposed the intersection between the "entanglement wedge" and the corresponding WDW patch for 'complexity = action' for mixed states constructed from subregions. It would be instructive to understand this proposal by considering more complicated examples.

Acknowledgements We would like to thank Mohsen Alishahiha, Amin Faraji-Astaneh and Mohammad H. Vahidinia for fruitful discussions and M. Reza Mohammadi-Mozaffar for careful reading of the manuscript.

Open Access This article is distributed under the terms of the Creative Commons Attribution 4.0 International License (http://creativecomm ons.org/licenses/by/4.0/), which permits unrestricted use, distribution, and reproduction in any medium, provided you give appropriate credit to the original author(s) and the source, provide a link to the Creative Commons license, and indicate if changes were made. Funded by SCOAP ${ }^{3}$.

\section{References}

1. S. Ryu, T. Takayanagi, Holographic derivation of entanglement entropy from AdS/CFT. Phys. Rev. Lett. 96, 181602 (2006). doi:10. 1103/PhysRevLett.96.181602. arXiv:hep-th/0603001

2. S. Ryu, T. Takayanagi, Aspects of holographic entanglement entropy. JHEP 0608, 045 (2006). doi:10.1088/1126-6708/2006/ 08/045. arXiv:hep-th/0605073

3. L. Susskind, Computational complexity and black hole horizons. Fortsch. Phys. 64, 24 (2016). doi:10.1002/prop.201500092. arXiv:1403.5695 [hep-th], arXiv:1402.5674 [hep-th]

4. D. Stanford, L. Susskind, Complexity and shock wave geometries. Phys. Rev. D 90(12), 126007 (2014). doi:10.1103/PhysRevD.90. 126007. [arXiv: 1406.2678 [hep-th]]

5. L. Susskind, Entanglement is not enough. Fortsch. Phys. 64, 49 (2016). doi:10.1002/prop.201500095. arXiv:1411.0690 [hep-th]

6. A.R. Brown, D.A. Roberts, L. Susskind, B. Swingle, Y. Zhao, Holographic complexity equals bulk action? Phys. Rev. Lett 116(19), 191301 (2016). doi:10.1103/PhysRevLett.116.191301. arXiv:1509.07876 [hep-th]

7. A.R. Brown, D.A. Roberts, L. Susskind, B. Swingle, Y. Zhao, Complexity, action, and black holes. Phys. Rev. D 93(8), 086006 (2016). doi:10.1103/PhysRevD.93.086006. arXiv:1512.04993 [hep-th]

8. O. Ben-Ami, D. Carmi, On volumes of subregions in holography and complexity. JHEP 1611, 129 (2016). doi:10.1007/ JHEP11(2016)129. arXiv:1609.02514 [hep-th]

9. J. Couch, W. Fischler, P. H. Nguyen, Noether charge, black hole volume and complexity. arXiv:1610.02038 [hep-th]

10. S. Chapman, H. Marrochio, R. C. Myers, complexity of formation in holography. arXiv:1610.08063 [hep-th]

11. D. Carmi, R. C. Myers, P. Rath, Comments on holographic complexity. arXiv:1612.00433 [hep-th]

12. L.Y. Hung, R.C. Myers, M. Smolkin, A. Yale, Holographic calculations of renyi entropy. JHEP 1112, 047 (2011). doi:10.1007/ JHEP12(2011)047. arXiv:1110.1084 [hep-th]

13. X. Dong, The gravity dual of renyi entropy. Nat. Commun. 7, 12472 (2016). doi:10.1038/ncomms12472. arXiv:1601.06788 [hep-th]

14. M. Miyaji, T. Numasawa, N. Shiba, T. Takayanagi, K. Watanabe, Distance between Quantum States and Gauge-Gravity Duality. Phys. Rev. Lett 115(26), 261602 (2015). doi:10.1103/PhysRevLett. 115.261602. arXiv:1507.07555 [hep-th]
15. D. Bak, Information metric and Euclidean Janus correspondence. Phys. Lett. B 756, 200 (2016). doi:10.1016/j.physletb.2016.03.012. arXiv: 1512.04735 [hep-th]

16. D. Momeni, S.A.H. Mansoori, R. Myrzakulov, Holographic complexity in gauge/string superconductors. Phys. Lett. B 756, 354 (2016). doi:10.1016/j.physletb.2016.03.031. arXiv:1601.03011 [hep-th]

17. N.S. Mazhari, D. Momeni, S. Bahamonde, M. Faizal, R. Myrzakulov, Holographic complexity and fidelity susceptibility as holographic information dual to different volumes in AdS. Phys. Lett. B 766, 94 (2017). doi:10.1016/j.physletb.2016.12.060. arXiv:1609.00250 [hep-th]

18. M. Alishahiha, Holographic complexity. Phys. Rev. D 92(12), 126009 (2015). doi:10.1103/PhysRevD.92.126009. arXiv: 1509.06614 [hep-th]

19. S. Banerjee, J. Erdmenger, D. Sarkar, Connecting fisher information to bulk entanglement in holography. arXiv:1701.02319 [hepth]

20. N. Lashkari, M. Van Raamsdonk, Canonical energy is quantum fisher information. JHEP 1604, 153 (2016). doi:10.1007/ JHEP04(2016)153. arXiv:1508.00897 [hep-th]

21. M. Alishahiha, A. Faraji Astaneh, A. Naseh, M. H. Vahidinia, On complexity for higher derivative gravities. arXiv:1702.06796 [hepth]

22. L. Lehner, R.C. Myers, E. Poisson, R.D. Sorkin, Gravitational action with null boundaries. Phys. Rev. D 94(8), 084046 (2016). doi:10.1103/PhysRevD.94.084046. arXiv:1609.00207 [hep-th]

23. M. Flory, A complexity/fidelity susceptibility g-theorem for $\mathrm{AdS}_{3} / \mathrm{BCFT}_{2}$. JHEP 1706, 131 (2017). doi:10.1007/ JHEP06(2017)131. arXiv:1702.06386 [hep-th]

24. H. Casini, M. Huerta, Universal terms for the entanglement entropy in 2+1 dimensions. Nucl. Phys. B 764, 183 (2007). doi:10.1016/j. nuclphysb.2006.12.012. arXiv:hep-th/0606256

25. H. Casini, M. Huerta, L. Leitao, Entanglement entropy for a Dirac fermion in three dimensions: Vertex contribution. Nucl. Phys. B 814, 594 (2009). doi:10.1016/j.nuclphysb.2009.02.003. arXiv:0811.1968 [hep-th]

26. T. Hirata, T. Takayanagi, AdS/CFT and strong subadditivity of entanglement entropy. JHEP 0702, 042 (2007). arXiv:hep-th/0608213

27. R.C. Myers, A. Singh, Entanglement entropy for singular surfaces. JHEP 1209, 013 (2012). doi:10.1007/JHEP09(2012)013. arXiv:1206.5225 [hep-th]

28. A. Singh, Holographic entanglement entropy: RG flows and singular surfaces. PhD thesis, University of Waterloo, 2012

29. P. Bueno, R.C. Myers, W. Witczak-Krempa, Universality of corner entanglement in conformal field theories. Phys. Rev. Lett. 115, 021602 (2015). doi:10.1103/PhysRevLett.115.021602. arXiv:1505.04804 [hep-th]

30. P. Bueno, R.C. Myers, Corner contributions to holographic entanglement entropy. JHEP 1508, 068 (2015). doi:10.1007/ JHEP08(2015)068. arXiv:1505.07842 [hep-th]

31. D.W. Pang, Corner contributions to holographic entanglement entropy in non-conformal backgrounds. JHEP 1509, 133 (2015). doi:10.1007/JHEP09(2015)133. arXiv:1506.07979 [hep-th]

32. M. Alishahiha, A.F. Astaneh, P. Fonda, F. Omidi, Entanglement entropy for singular surfaces in hyperscaling violating theories. JHEP 1509, 172 (2015). doi:10.1007/JHEP09(2015)172. arXiv:1507.05897 [hep-th]

33. R.X. Miao, A holographic proof of the universality of corner entanglement for CFTs. JHEP 1510, 038 (2015). doi:10.1007/ JHEP10(2015)038. arXiv:1507.06283 [hep-th]

34. P. Bueno, R.C. Myers, W. Witczak-Krempa, Universal corner entanglement from twist operators. JHEP 1509, 091 (2015). doi:10. 1007/JHEP09(2015)091. arXiv:1507.06997 [hep-th] 
35. P. Bueno, R.C. Myers, Universal entanglement for higher dimensional cones. JHEP 1512, 168 (2015). doi:10.1007/ JHEP12(2015)168. arXiv:1508.00587 [hep-th]

36. R.C. Myers, A. Singh, Comments on holographic entanglement entropy and RG flows. JHEP 1204, 122 (2012). doi:10.1007/ JHEP04(2012)122. arXiv:1202.2068 [hep-th]

37. M.R. Mohammadi Mozaffar, A. Mollabashi, F. Omidi, Holographic mutual information for singular surfaces. JHEP 1512, 082 (2015). doi:10.1007/JHEP12(2015)082. arXiv:1511.00244 [hepth]
38. P. Bueno, W. Witczak-Krempa, Bounds on corner entanglement in quantum critical states. Phys. Rev. B 93, 045131 (2016). doi:10. 1103/PhysRevB.93.045131. arXiv:1511.04077 [cond-mat.str-el]

39. P. Bueno, V. S. Min, A. J. Speranza, M. R. Visser, Entanglement equilibrium for higher order gravity. arXiv:1612.04374 [hep-th] 